\title{
Unified Models for Coupled Inductors Applied to Multiphase PWM Converters
}

\author{
Minjie Chen, Senior Member, IEEE, Charles R. Sullivan, Fellow, IEEE
}

\begin{abstract}
Circuit models for multiphase coupled inductors are summarized, compared, and unified. Multiwinding magnetic structures are classified into parallel-coupled structures and series-coupled structures. For parallel-coupled structures used for multiphase inductors, the relationships between a) inductancematrix models, b) extended cantilever models, c) magnetic-circuit models, d) multiwinding transformer models, e) gyrator-capacitor models, and f) inductance-dual models are examined and discussed. These models represent identical physical relationships in the multiphase coupled inductors, but emphasize different physical aspects and offer distinct design insights. The circuit duality between the series-coupled structure and the parallel-coupled structure is explored. Design equations for interleaved multiphase buck converters based on these models are streamlined and summarized, and a simplified equation showing the relationships between current ripple with and without coupling is presented. The models and design equations are verified through theoretical derivation, SPICE simulation, and experimental measurements.
\end{abstract}

Index Terms-lumped circuit model, coupled inductor, multiphase buck converter, magnetic-circuit model, multi-winding transformer, interphase transformer, inductance-dual model.

\section{INTRODUCTION}

$\mathbf{M}$ ULTIPHASE coupled inductors are widely used in many power electronics applications [1]-[20]. Particularly in interleaved multiphase PWM converters, they can improve the efficiency, enhance the functionality, minimize the energy storage, reduce the passive component size, avoid saturation, and improve the transient response. Designing high performance power converters with multiphase coupled inductors requires advanced models and tools.

Models for multiphase coupled inductors can be classified into two categories: 1) Math-based models focus on the mathematical coupling relationships between windings. Math-based models are derived based on the inductance matrix, reluctance matrix, or permeance matrix. Information about the core geometry, material properties, and winding structure is not explicitly included. Math-based models can be represented by a few convenient circuit models whose element values may not have explicit physical meanings, such as the classic $T$ model, $\pi$ model, star model, delta model, and extended cantilever model [21]-[28]. 2) Physics-based models represent the physical geometry of the magnetic structure more directly. Each portion of the magnetic structure is represented by a lumped circuit

Minjie Chen is with the Department of Electrical and Computer Engineering and Andlinger Center for Energy and the Environment at Princeton University, Princeton, NJ 08540, USA.

Charles R. Sullivan is with the Thayer School of Engineering of Dartmouth College, Hanover, NH 03755, USA.

This work was supported by the National Science Foundation under CAREER Award \#1847365 and the Princeton SEAS Innovation Fund.

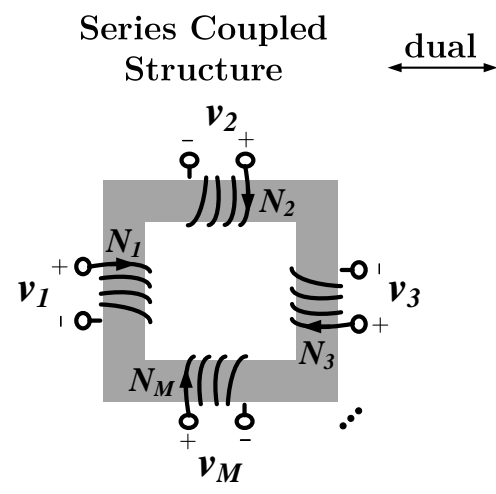

Parallel Coupled
Structure

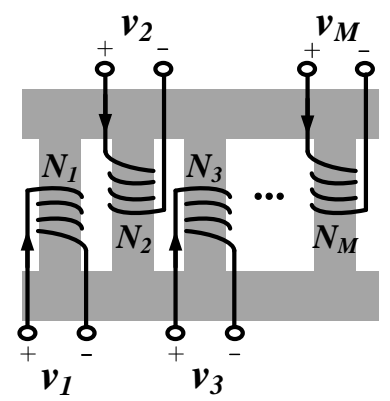

Fig. 1. Two major categories of multiwinding magnetic structures: 1) seriescoupled structure; and 2) parallel-coupled structure.

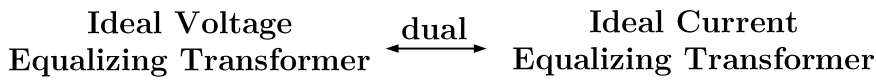

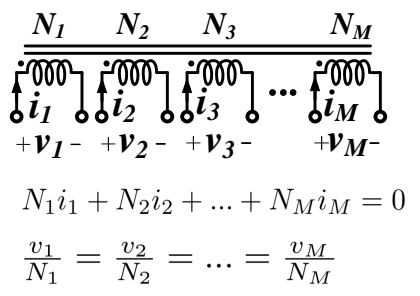

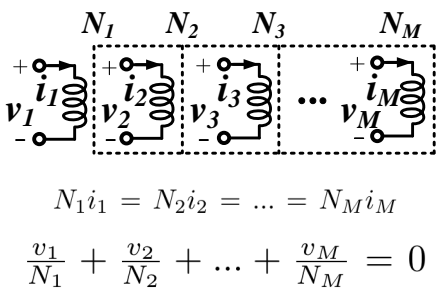

Fig. 2. Symbols and equations for: 1) ideal voltage equalizing transformer; and 2) ideal current-equalizing transformer [29]. With infinite core permeability and negligible leakage, a series-coupled structure can be modeled as an ideal voltage equalizing transformer, and a parallel-coupled structure can be modeled as an ideal current-equalizing transformer. For the ideal currentequalizing transformer, we show the core topology with dashed lines, and label winding polarity with arrows rather than dots, indicating the direction of current that is equal in the corresponding current equation, just as the dots indicate the direction of voltage that is equal in the corresponding voltage equation for the voltage equalizing transformer.

element, and many lumped circuit elements are combined into a complete circuit. The reluctance circuit model, the gyratorcapacitor model, the inductance-dual model, and the modular multi-layer model are physics-based models [30]-[39]. Finiteelement models are also physics-based models.

In their basic, linear form, all models discussed in this paper are equivalent. However, different models offer different design insights. Math-based models are well suited to theoretical analysis of power converters, especially if the magnetic components are already designed. Physics-based models offer the oppportunity for natural extensions to capture core loss, saturation, and the details of the flux distribution in the core. Designers should choose an appropriate model based on 
the information that is needed in the design process. Often, moving from math-based models to physics-based models can give experienced circuit designers useful insight on circuit operation that is harder to get from math-based models.

Fig. 1 shows two major categories of multiwinding magnetic structures: 1) Series-coupled structures, in which the flux paths of multiple windings are configured in series; 2) Parallel-coupled structures, in which the flux paths of multiple windings are configured in parallel. Fig. 2 shows their circuit symbols. With zero leakage flux, the series-coupled structure forces the magnetic flux $\Phi$ of all windings to be the same, leading to a bonded voltage relationship across windings. With infinite permeability, the parallel-coupled structure forces the magneto-motive-force (MMF) of all windings to be the same, leading to a bonded current relationship across windings. The series-coupled structure functions as an ideal voltage equalizing transformer in which $N_{1} i_{1}+N_{2} i_{2}+\ldots+N_{M} i_{M}=0$, and $\frac{v_{1}}{N_{1}}=\frac{v_{2}}{N_{2}}=\ldots=\frac{v_{M}}{N_{M}}$; the parallel-coupled structure functions as an ideal current-equalizing transformer in which $N_{1} i_{1}=N_{2} i_{2}=\ldots=N_{M} i_{M}$, and $\frac{v_{1}}{N_{1}}+\frac{v_{2}}{N_{2}}+\ldots+\frac{v_{M}}{N_{M}}=0$.

The goal of this paper is to investigate theoretical frameworks that are most practical for modeling sophisticated coupled magnetic structures and their application in multiphase PWM converters. The series-coupled structure is well studied in the literature [26]-[28], [40]-[47]. Its mathematical symbol, an ideal voltage equalizing transformer, is commonly supported by commercial SPICE simulation platforms. The parallel-coupled structure is less commonly found in power electronics applications. Moreover, ideal multi-winding current-equalizing transformers are not well supported in mainstream SPICE simulation platforms. A majority of this paper focuses on models for parallel-coupled structures. We summarize options for model structure and show how to use these models to analyze and simulate buck converters with multiphase coupled inductors, including developing a simplified equation showing the relationships between current ripple for interleaved multiphase buck converters with and without coupling.

The remainder of this paper is organized as follows: Section II introduces general models for multiwinding structures. Section III simplifies these models for idealized multiwinding coupled inductors. Section IV presents models for ladder-core coupled inductors and discusses the relationship between this model and a ladder model for a layered multiwinding transformer. Section $\mathrm{V}$ unifies these models and develops calculations to convert parameters between them. Section VI applies the models to idealized coupled inductors in multiphase buck converters and provides design guidelines. Section VII verifies these models, and presents simulation and experimental results of a multiphase coupled inductor buck converter. After Section VIII concludes the paper, appendices provide background on effective inductance parameters, sometimes used to describe circuit operation, and on extracting model parameters from measurements.

\section{Generalized Multiwinding Structures}

The voltage and current of an arbitrary multiphase coupled inductor is described by an inductance matrix $\mathbf{L}$ :

$$
\underbrace{\left[\begin{array}{c}
v_{1} \\
v_{2} \\
\vdots \\
v_{M}
\end{array}\right]}_{\mathbf{V}}=\underbrace{\left[\begin{array}{cccc}
L_{11} & L_{12} & \cdots & L_{1 M} \\
L_{21} & L_{22} & \cdots & L_{2 M} \\
\vdots & \vdots & \ddots & \vdots \\
L_{M 1} & L_{M 2} & \cdots & L_{M M}
\end{array}\right]}_{\mathbf{L}} \underbrace{\left[\begin{array}{c}
\frac{d i_{1}}{d t} \\
\frac{d i_{2}}{d t} \\
\vdots \\
\frac{d i_{M}}{d t}
\end{array}\right]}_{\frac{d \mathbf{I}}{d t}} .
$$

Here $\mathbf{V}$ and $\mathbf{I}$ are the voltage and current of the $M$ windings. The inductance matrix is a symmetric matrix with positive and negative element values which are the self and mutual inductances. It describes the mathematical coupling relationship between windings and is applicable to all linear multiwinding coupled magnetics, neglecting losses. The element values of this matrix can be identified by physical modeling such as finite-element analysis or by experimental measurements. Fig. 3 shows an example four-winding magnetic structure with a combination of series, parallel, and air-gap configurations. Fig. 4 shows the inductance-matrix model of this example [21]-[24]. Many SPICE simulation platforms, e.g., PSIM, LTSpice and Simplis, support the use of the inductance-matrix model. A similar model which decouples the mutual coupling relationships is the extended cantilver model [26] as shown in Fig. 5. In an extended cantilever model, all elements are non-coupled inductors and ideal transformers.

Both the inductance-matrix model and the extended cantilever model are math-based models. One drawback of mathbased models is that the geometry and material property information is not explicitly shown in the model. Limited insight on magnetic structure design is offered. Physics-based models can offer more direct insight on the relationship between the physical structure and the model, and are considered next.

The magnetic-circuit model (reluctance circuit model) [21] [24] as shown in Fig. 6 is one of the most widely used physicsbased models. Each portion of the magnetic core is modeled as a reluctance. Each winding is modeled as an MMF source driving the reluctance circuit as a voltage source. The throughvariable is the magnetic flux $\Phi$, and the across variable is the MMF, $\mathcal{F}$. The magnetic-circuit model is a static model.

One limitation of the magnetic-circuit model is that it is cumbersome to simulate in SPICE. In a SPICE model, the magnetic circuit is constructed using across and through variables of current and voltage to represent MMF and flux. To model the interaction between this magnetic circuit and the electric circuit connected to the windings, on must consider that the current is linearly related to $\operatorname{MMF}(\mathcal{F})$, and the voltage to the derivative of flux $(d \Phi / d t)$. Added circuits are needed to implement the linear scaling and time-derivative/integral relationships which are critical in dynamic simulations [39].

The gyrator-capacitor model differs from the magneticcircuit model by replacing the through variable $\Phi$ with its time-derivative $\dot{\Phi}=d \Phi / d t$, replacing the reluctances with capacitors whose capacitance equal to the permeance $\mathcal{P}=1 / \mathcal{R}$, and replacing the MMF sources with gyrators which convert current into voltage [31], [32]. Fig. 7 shows an example gyrator-capacitor model derived from the magnetic-circuit 


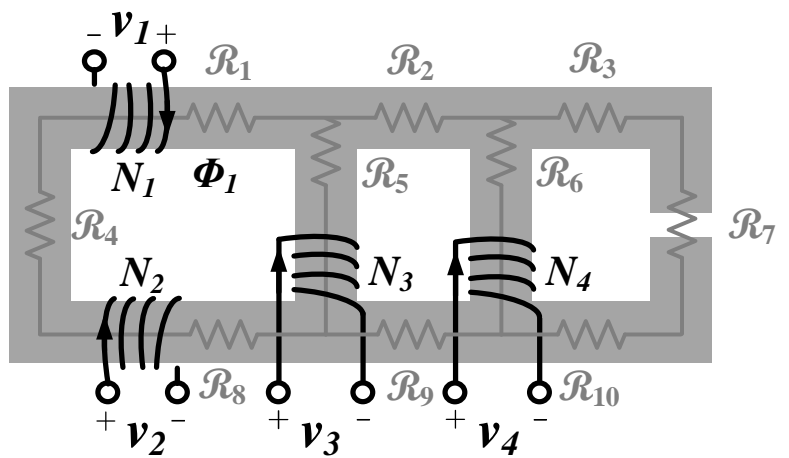

Fig. 3. An example four-winding magnetic structure with a combination of series, parallel, and air-gap configurations.

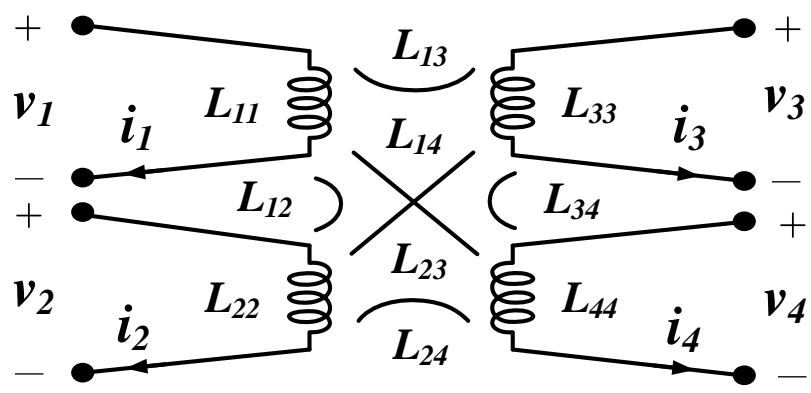

Fig. 4. Inductance-matrix model of the magnetic structure in Fig. 3.

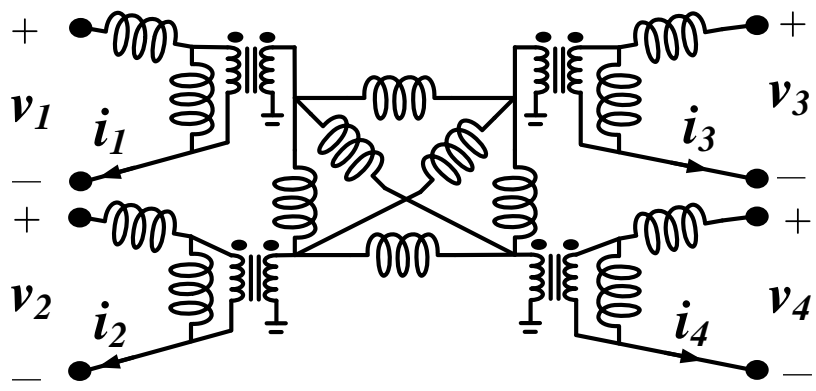

Fig. 5. Extended cantilever model of the magnetic structure in Fig. 3.

model in Fig. 6. The two lumped circuit models share the same network structure but have different component values. In the gyrator-capacitor model, the through variable has units of $\mathrm{V}$, and the across variable has units of $\mathrm{A}$.

It is sometimes beneficial to apply topological duality [48]-[51] to the gyrator-capacitor model and create an inductance-dual model [33]-[38], as illustrated in Fig. 8. In the inductance-dual model, the elements representing the magnetic core sections are inductors with inductance values equal to the permeance values $\mathcal{P}=1 / \mathcal{R}$, in units of henry $(\mathrm{H})$. The through variable is current, and the across variable is voltage. The terminals of the model are ideal transformers with turns ratios equal to the physical turns of the windings.

All models represent the same math and physics when applied in the linear forms presented in this section. However, they all have strengths and weakness, as listed in Table I, and as further developed in the remainder of the paper.

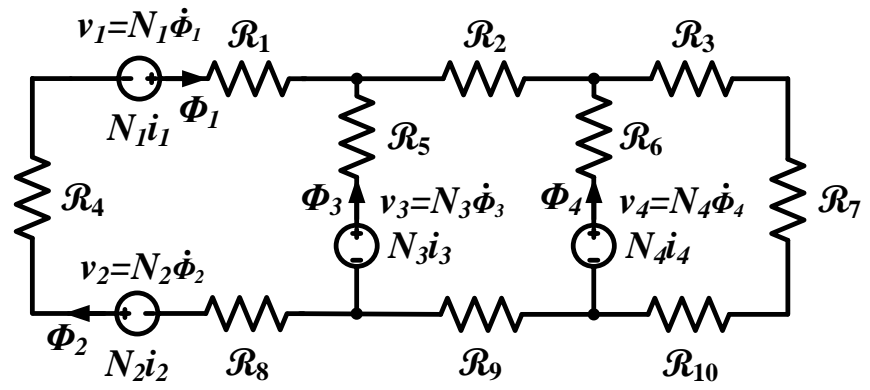

Fig. 6. Magnetic-circuit model of the magnetic structure in Fig. 3.

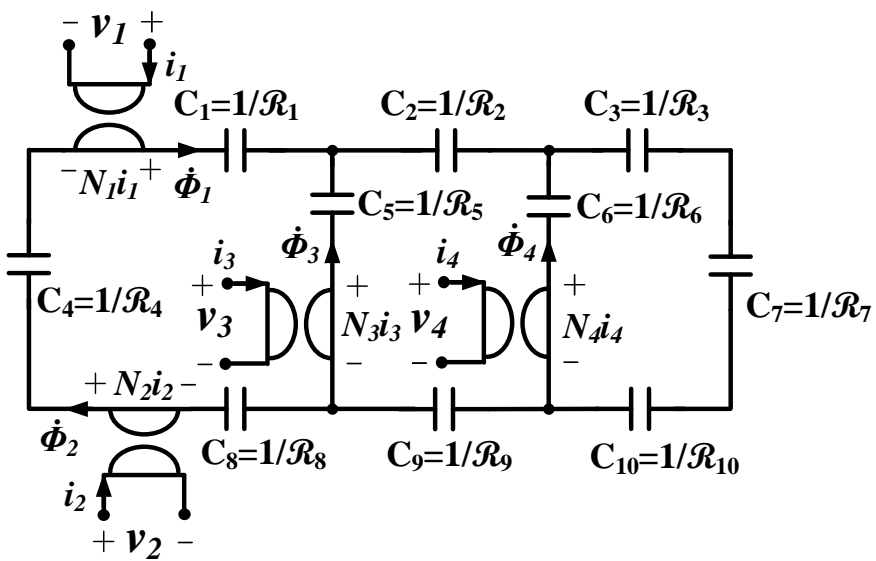

Fig. 7. Gyrator-capacitor model of the magnetic structure in Fig. 3.

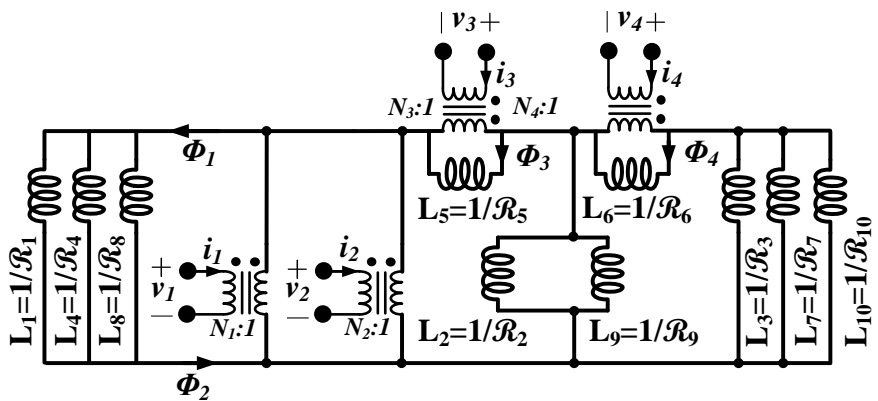

Fig. 8. Inductance-dual model of the magnetic structure in Fig. 3.

\section{IdEALIZED Multiphase COUPLED Inductors}

In this section, we apply the general modeling approaches discussed in Section II to the idealized multiwinding coupled inductor structure shown in Fig. 9, with $M$ outer legs and one leg in the center. This is a parallel-coupled multiwinding structure with a symmetric geometry. Each of the outer legs is encircled by an $N$-turn winding. High permeability magnetic materials are used, and a gap is used in the center leg to control the inductance and avoid saturation with balanced dc currents. Optional small gaps are sometimes used in the outer legs to avoid saturation with small imbalances in the dc currents.

Fig. 10 shows the magnetic-circuit model of this parallel structure. The model comprises $M$ outer leg reluctances $\mathcal{R}_{L}$, and one center leg reluctance $\mathcal{R}_{C}$. Each outer leg is driven by an MMF source $\mathcal{F}=N i$. Fig. 11 shows the gyratorcapacitor model of this structure, following the same changes in variables as with Figs. 6 and 7. 
TABLE I

GENERAL ADVANTAGES AND DISADVANTAGES OF MODEL TYPES

\begin{tabular}{|c|c|c|}
\hline & Advantages & Disadvantages \\
\hline $\begin{array}{l}\text { Inductance-Matrix } \\
\text { Model }\end{array}$ & $\begin{array}{l}\text { - Standard for mathematical analysis. } \\
\text { - Natural for state space circuit models. }\end{array}$ & - No explicit connection with physical structure. \\
\hline Transformer Models & $\begin{array}{ll}- & \text { Familiar to circuit designers. } \\
\text { - } & \text { Simplified models for ideal cases elucidate circuit operation. }\end{array}$ & - No explicit connection with physical structure. \\
\hline $\begin{array}{l}\text { Magnetic-Circuit } \\
\text { Model }\end{array}$ & $\begin{array}{l}\text { Widely used for physical design } \\
\text { - Most straightforward connection to physical structure. }\end{array}$ & $\begin{array}{l}\text { - Use in circuit simulation requires extra dynamic } \\
\text { blocks. }\end{array}$ \\
\hline $\begin{array}{l}\text { Gyrator-Capacitor } \\
\text { Model }\end{array}$ & $\begin{array}{l}\text { - } \quad \text { Circuit directly represents dynamics and energy storage. } \\
\text { - Topology matches structure. }\end{array}$ & $\begin{array}{l}\text { - Use in circuit simulation requires gyrators. } \\
\text { - } \quad \text { Relationship to physical structure is abstract. }\end{array}$ \\
\hline $\begin{array}{l}\text { Inductance-Dual } \\
\text { Model }\end{array}$ & $\begin{array}{l}\text { - Can be used directly in circuit simulation. } \\
\text { - Circuit directly represents dynamics and energy storage. } \\
\text { - } 1: 1 \text { correspondence between circuit elements and physical ele- } \\
\text { ments. }\end{array}$ & $\begin{array}{l}\text { - The circuit topology is the topological dual of the } \\
\text { physical reluctance path. } \\
\text { Representing DC flux density accurately in simu- } \\
\text { lation requires DC transformers and extra resistors. }\end{array}$ \\
\hline
\end{tabular}

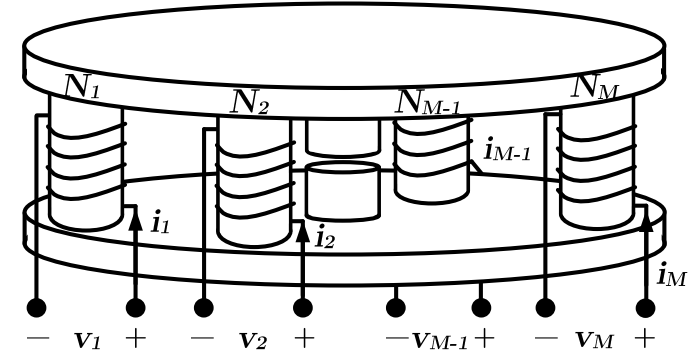

Fig. 9. A symmetric multiphase coupled inductor with many windings. This structure has $M$ outer legs and a center leg.

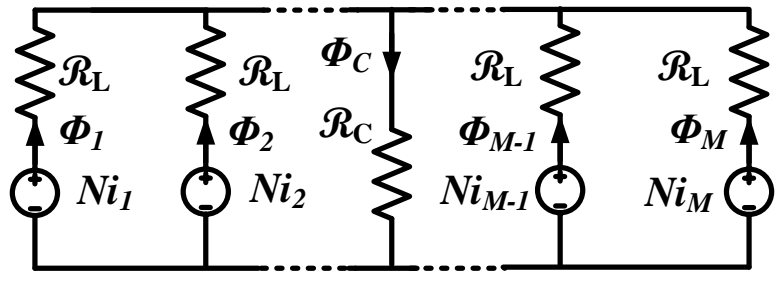

Fig. 10. Magnetic-circuit model of the structure in Fig. 9. All windings have $N$ turns. The reluctances of the outer legs are $\mathcal{R}_{L}$, and the reluctance of the center leg is $\mathcal{R}_{C}$. This structure is fully symmetric.

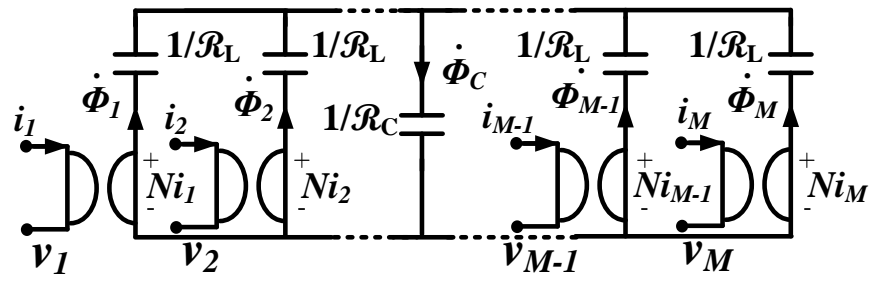

Fig. 11. Gyrator-capacitor model of the structure in Fig. 9. All windings have $N$ turns. Labeled values are capacitance values. The across variable is $\mathcal{F}$; the through variable is $\dot{\Phi}=d \Phi / d t$.

Finding the topological dual of the magnetic circuit or the gyrator-capacitor model results in the inductance-dual model shown in Fig. 12. To capture the dc bias of the magnetic core in circuit simulations, the ideal transformers in the inductancedual model should operate in dc, i.e., the transformer current relationship should apply to dc as well as ac currents. However,

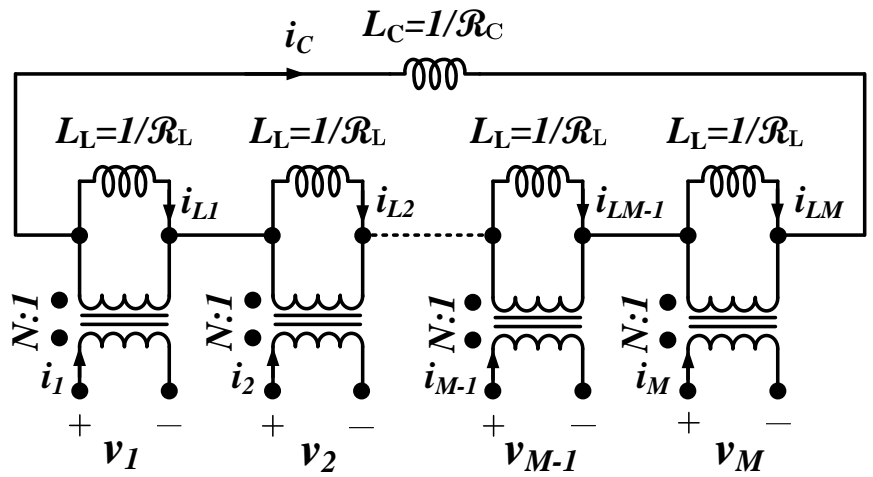

Fig. 12. Inductance-dual model of the structure in Fig. 9. $1 / \mathcal{R}_{C}$ and $1 / \mathcal{R}_{L}$ represent the inductive elements of the center leg and the outer legs, respectively. In SPICE simulations with ideal transformers, $i_{C}$ and $i_{L}$ are linearly related to $\Phi_{C}$ and $\Phi_{L}$ in the center leg and the outer legs, respectively.

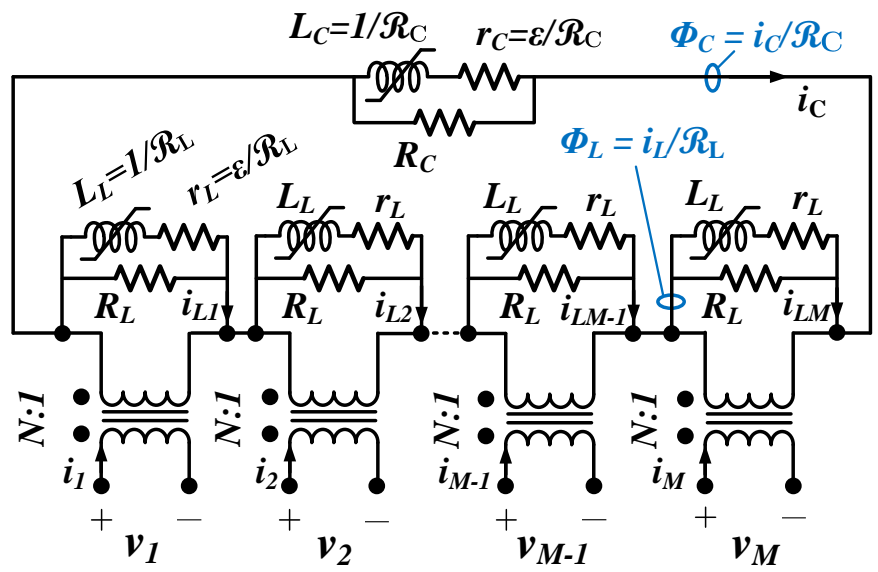

Fig. 13. Inductance-dual model including saturable inductors and core-loss resistors. The series resistors $r_{L}$ and $r_{C}$ are used to ensure the correct dc current. The parallel resistors $R_{L}$ and $R_{C}$ capture the core loss. By probing the current in the inductance-dual model and dividing it by the reluctance value, on can visualize the magnetic flux in each portion of the core and, for example, see saturation effects. In a magnetic-circuit model, $\frac{1}{2} \Phi^{2} \mathcal{R}$ is the energy storage. In an inductance-dual model, $\frac{1}{2} i^{2} / \mathcal{R}$ is the energy storage.

this is insufficient to make the the model in Fig. 12 capture the dc bias level in each core path, because there is a loop of ideal inductors for which the dc current is undetermined. 


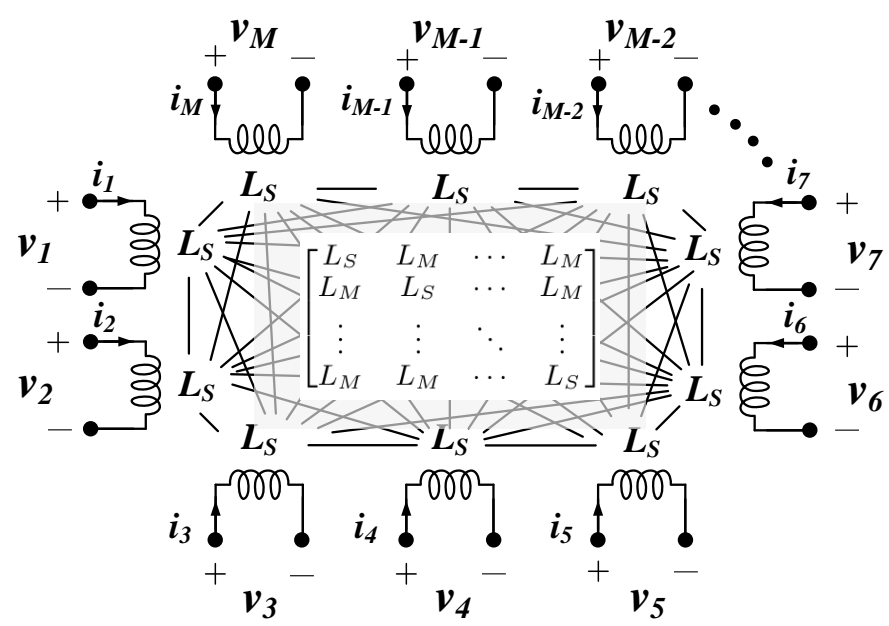

Fig. 14. Inductance-matrix model of the structure in Fig. 9. The element values of this model come from the inductance matrix.

To solve this problem, the model in Fig. 13 includes a small, non-physical resistance in series with each inductor $\left(r_{L}\right)$.

For proper dc balancing, the resistance of each is proportional to, but orders of magnitude smaller than, the impedance of the inductor it is connected in series with. Specifically, to ensure that the losses in this dc balancing resistor are negligible, its losses at the switching frequency should be much smaller than the core loss at the switching frequency. These core losses, for a given core segment $x$, could be modeled by a series resistor of value $r_{c, x}=\omega_{S} /\left(Q_{\text {core }} \mathcal{R}_{x}\right)$, where $Q_{\text {core }}$ is the quality factor of the core material at this frequency. So if we choose a dc-balancing resistor for segment $x$ such that $r_{x} \ll \omega_{S} /\left(Q_{x} \mathcal{R}_{x}\right)$, it will incur much less loss than the core. For leg with an air gap, the effective quality factor is $Q_{x}$ is increased by the ratio of the reluctances in that leg, to $Q_{x}=Q_{\text {core }} \mathcal{R}_{\text {gap }, x} / \mathcal{R}_{\text {core }, x}$, as the energy stored in the air gap has no loss. Because we need the same proportionality for each segment for proper balancing, we choose $r_{x}$ as

$$
r_{x}=\frac{\omega_{S}}{100 Q_{\max } \mathcal{R}_{x}},
$$

where $Q_{\max }$ is the maximum $Q$ among all the legs, and the factor of 100 is a large enough factor to ensure that the losses are negligible.

With the flux, including dc components, represented by inductor currents, one can probe the currents $i_{L}(t)$ and $i_{C}(t)$, divide them by the corresponding reluctances $\mathcal{R}_{L}$ and $\mathcal{R}_{C}$, and visualize the magnetic fluxes $\Phi_{L}(t)$ and $\Phi_{C}(t)$ in circuit simulations. Fig. 13 also shows an example of a way to implement core loss and saturation effects in the inductancedual model. Each portion of the magnetic core is implemented as a saturable inductor $\left(L_{L}\right.$ or $\left.L_{C}\right)$. An additional resistor is then connected in parallel with the saturable inductor and resistor to capture the core loss of each portion of the magnetic core. This resistance is usually non-linear. Nonlinear core behavior can also be captured in a magnetic circuit model, as used in [17] to model saturation effects in coupled indcutors.

Fig. 14 illustrates the concept of an inductance-matrix model of a multiwinding coupled inductor. The core of this model is

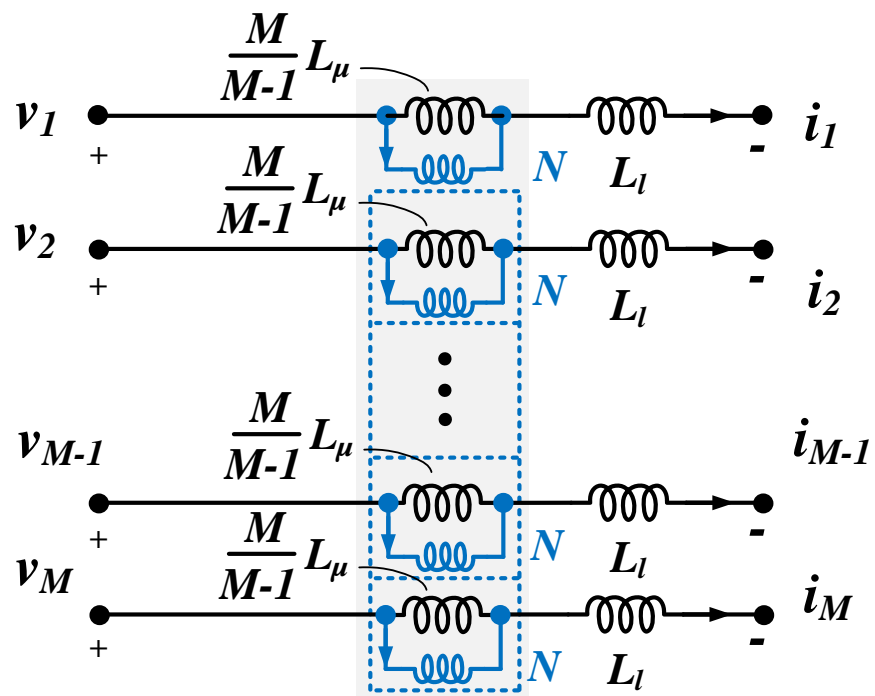

Fig. 15. Multiwinding transformer model of the structure in Fig. 9 implemented with an ideal current-equalizing transformer (in blue). The ideal current-equalizing transformer equalizes the MMFs of all blue windings and forces the sum of the per- turn voltages of all windings to be zero.

a $M \times M$ inductance matrix as per (1). The inductance-matrix model is interchangeable with a multiwinding transformer model, as well as with all the other models under discussion.

There are many ways to implement a multiwinding transformer model. Fig. 15 shows one example using an ideal current-equalizing transformer. This model includes a magnetizing inductance in parallel with each each winding of the ideal current-equalizing transformer and a leakage inductance $L_{l}$ in series. The turns ratio of the ideal current-equalizing transformer is $\{1: 1: \ldots: 1\}$, assuming equal numbers of turns $N$ in each physical winding. The individual magnetizing inductances have a value $\frac{M}{M-1} L_{\mu}$, where $L_{\mu}$ is defined by analogy to magnetizing inductance in a two-winding transformer as $L_{\mu}=L_{S}-L_{l}$ where $L_{S}$ is the self inductance (measured on one winding of the structure with the others open-circuited) and is also equal to the self inductance in the inductance matrix equation. To write this model in the form of an inductance matrix, we also need the mutual inductance $L_{M}=-\frac{1}{M-1} L_{\mu}$.

Fig. 16 shows another implementation of a multiwinding transformer model using $M$ ideal voltage equalizing transformers. Each of these transformers has a turns ratio of $\{(M-1): 1: \ldots: 1\}$ (assuming all physical windings have the same number of turns). The magnetizing inductance reflected on the $\{M-1\}$ turn side is $\frac{M-1}{M} L_{\mu}$. The leakage inductance of each winding is $L_{l}$. More discussion of multiwinding transformer models is provided in [10].

The inductance-matrix model, the extended cantilever model, and the multiwinding transformer models are mathbased models. The magnetic-circuit model, the gyratorcapacitor model, and the inductance-dual model are physicsbased models. Math-based models describe the mathematical equations at the interface, from port-to-port. Physics-based models illustrate the physical behaviors of the magnetic core and the windings. These models are all interchangeable under 


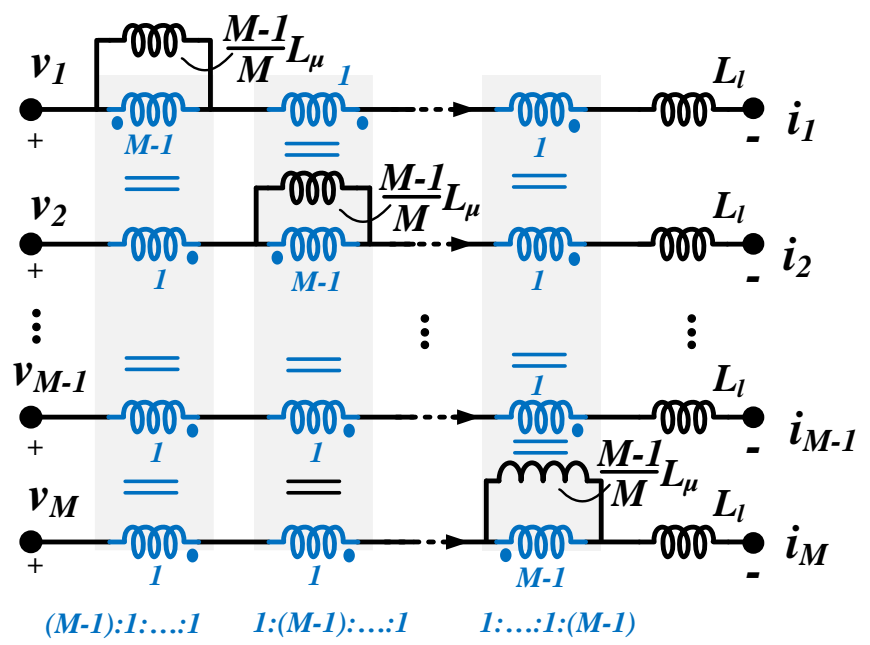

Fig. 16. Multiwinding transformer model of the structure in Fig. 9 implemented with an ideal voltage equalizing transformer (in blue). The ideal voltage equalizing transformer equalizes the per-turn voltages of all blue windings and forces the sum of the MMFs of all windings to be zero.

the same set of assumptions, but have advantages and disadvantages as described in Table I. In particular, the transformer model in Fig. 15 is advantageous for understanding circuit operation, and the inductance-dual model in Fig. 12 offers easy application in circuit simulations while retaining the ability to directly model the flux in individual magnetic circuit elements, and it can easily be extended to address non-ideal magnetic material behavior.

\section{LADDER-CORE AND LAYERED-WINDING STRUCTURES}

In this section, we first consider a commonly used coupled inductor structure, the ladder core, and relax the assumption that the top and bottom plates have negligible reluctance to develop a more accurate model. Then we consider a conventional multi-winding transformer with layered windings, to illustrate the topological dual relationship between the two structures.

\section{A. Ladder-Core Structure}

Fig. 17 shows a parallel-coupled structure implemented with a ladder core [1]-[5]. This ladder-core structure is attractive in designing coupled inductors for multiphase buck converters. If the reluctance of the top and bottom bars of this structure are neglected, any of the models previously discussed can be used. But unless they are very thick and/or have very high permeability, their reluctance may be significant, and the magnetic-circuit model in Fig. 18 applies. Although there is no explicit leakage path shown in Fig. 17, the flux leakage between the top and bottom bar is important to the operation of this structure in a typical circuit, and is modeled by the four leakage reluctances, $\mathcal{R}_{l}$. Fig. 19 shows the corresponding inductance-dual model. Based on Fig. 18, the magnetic fluxes $\left[\Phi_{1}, \ldots, \Phi_{M}\right]^{T}$ are functions of $\left[\mathcal{F}_{1}, \ldots, \mathcal{F}_{M}\right]^{T}$, which are the MMF values at the nodes labeled in Fig. 18. The magnetic

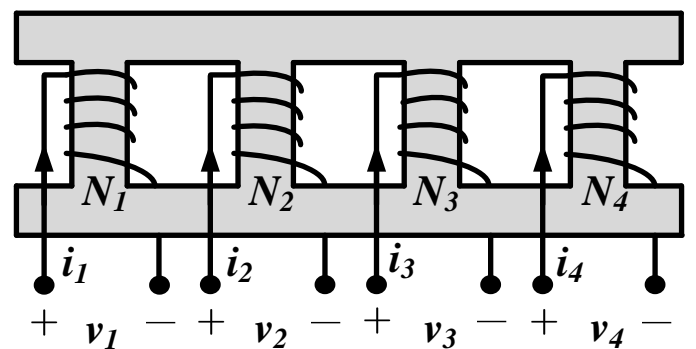

Fig. 17. Multiwinding coupled inductor with a ladder core. The windings' magnetic paths are in parallel and thus have approximately equal MMF and per-turn voltages that sum to zero.

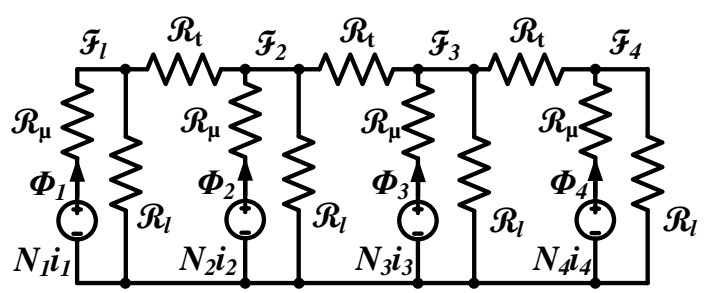

Fig. 18. Magnetic-circuit model of the ladder structure in Fig. 17. Labeled values $\mathcal{R}$ are reluctance values.

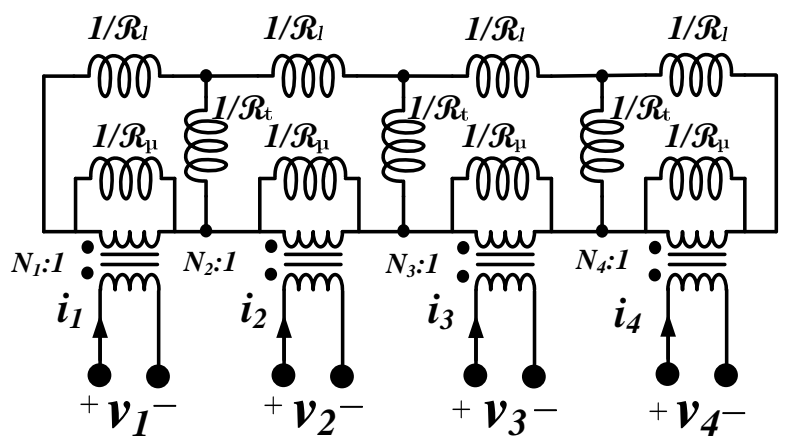

Fig. 19. Inductance-dual model of the ladder structure in Fig. 17. Labeled values $1 / \mathcal{R}$ are inductance values.

fluxes and MMFs are linked by a $M \times M$ permeance matrix $\mathbf{P}$ with element values determined by reluctance $\mathcal{R}_{l}$ and $\mathcal{R}_{t}$ :

$$
\underbrace{\left[\begin{array}{c}
\Phi_{1} \\
\Phi_{2} \\
\vdots \\
\Phi_{M}
\end{array}\right]}_{\boldsymbol{\Phi}}=\underbrace{\left[\begin{array}{cccc}
\frac{1}{\mathcal{R}_{t}}+\frac{1}{\mathcal{R}_{l}} & -\frac{1}{\mathcal{R}_{t}} & \cdots & 0 \\
-\frac{1}{\mathcal{R}_{t}} & \frac{1}{\mathcal{R}_{t}}+\frac{1}{\mathcal{R}_{l}} & \cdots & 0 \\
\vdots & \vdots & \ddots & \vdots \\
0 & 0 & \cdots & \frac{1}{\mathcal{R}_{t}}+\frac{1}{\mathcal{R}_{l}}
\end{array}\right]}_{\mathbf{P}} \underbrace{\left[\begin{array}{c}
\mathcal{F}_{1} \\
\mathcal{F}_{2} \\
\vdots \\
\mathcal{F}_{M}
\end{array}\right]}_{\mathbf{F}} .
$$

Using $\Phi_{x}=\frac{N i_{x}-\mathcal{F}_{x}}{\mathcal{R}_{\mu}}$, the vector of current in all windings (I) is a function of the vector of fluxes $(\boldsymbol{\Phi})$ in all legs and the permeance matrix $(\mathbf{P})$ :

$$
\mathbf{I}=\frac{1}{N}\left(\mathcal{R}_{\mu}+\mathbf{P}^{-1}\right) \boldsymbol{\Phi} .
$$

Taking the derivative of (4) results in the inductance matrix relationship connecting the winding current vector (I) and the winding voltage vector $(\mathbf{V})$ :

$$
\frac{d \mathbf{I}}{d t}=\frac{1}{N^{2}}\left(\mathcal{R}_{\mu}+\mathbf{P}^{-1}\right) \mathbf{V} .
$$




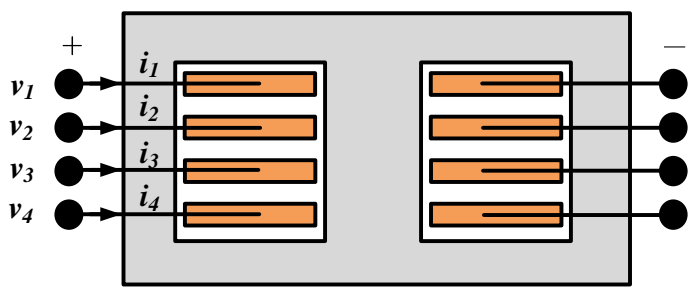

Fig. 20. Multiwinding planar transformer. Windings all encircle the same leg and are thus in series in the magnetic path. This series mangetic coupling results in approximately equal per-turn voltage and zero summed MMF.

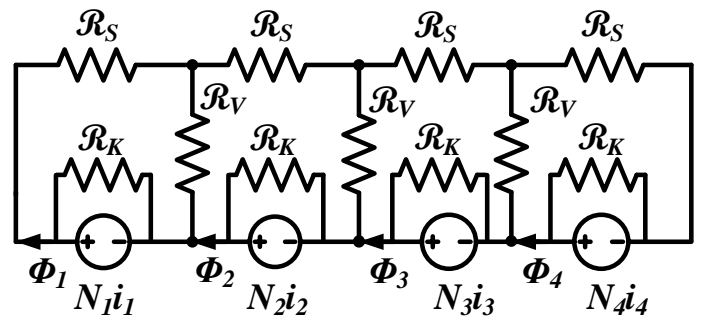

Fig. 21. Magnetic-circuit model of layered transformers such as the planar structure in Fig. 20. Labeled values are reluctance values.

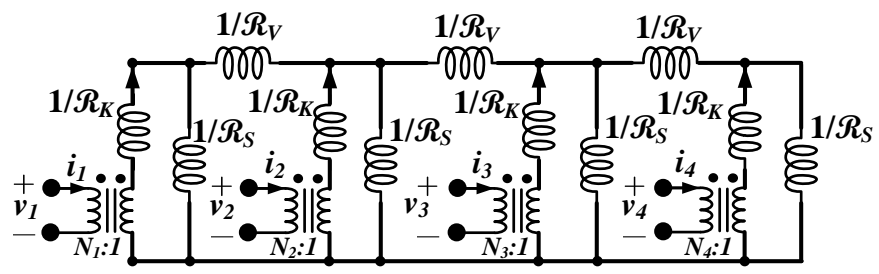

Fig. 22. Inductance-dual model of a layered transformer such as the planar structure in Fig. 20. Labeled values are inductance values. The inductancedual model was presented as a modular layer model in [41].

\section{B. Layered Winding Structure}

In this subsection, we consider a conventional multiwinding transformer with layered windings. These may be concentric wire- or foil-wound windings or stacked PCB windings, as shown in Fig. 20. Fig. 20 also shows a general schematic of this type of structure with multiple windings coupled to a single flux path. The corresponding magnetic-circuit and inductance-dual models of the layered structure, including a leakage path between each winding layer represented by $\mathcal{R}_{K}$, are shown in Figs. 21-22, respectively. The inductance-dual model of Fig. 22 can be further extended to cover skin and proximity effects [41].

A duality is observed between the ladder-core models and the layered winding models. The ladder-core structure has MMF sources in parallel, and the layered winding structure has MMF sources in series. The ladder-core structure requires windings to have similar MMFs, and the layered winding structure requires windings to have similar fluxes. The laddercore structure is usually used to couple windings to make their ac currents match more closely, and the layered winding structure is usually used to couple windings to achieve similar voltage per turn. The ladder-core structure functions well for coupled inductors in multiphase buck converters, and the layered winding structure functions well for multiport dc-dc converters, such as multi-active-bridge (MAB) converters and energy routers [45]-[47].

\section{UNIFying MOdELS FOR IDEALIZED STRUCTURES}

This section unifies and compares simplified models for coupled inductors in which the top and bottom plate or bar reluctance is neglected and the model is symmetric. The equation which describes the inductance-matrix model in Fig. 14 is (1). The multiwinding transformer models in Fig. 15 and Fig. 16 correspond to (1) with $L_{S}=L_{\mu}+L_{l}$ and $L_{M}=-\frac{1}{M-1} L_{\mu}$. The magnetic-circuit model in Fig. 10 can be described by a reluctance matrix $\mathbf{R}$ based on $\mathcal{R}_{L}$ and $\mathcal{R}_{C}$ :

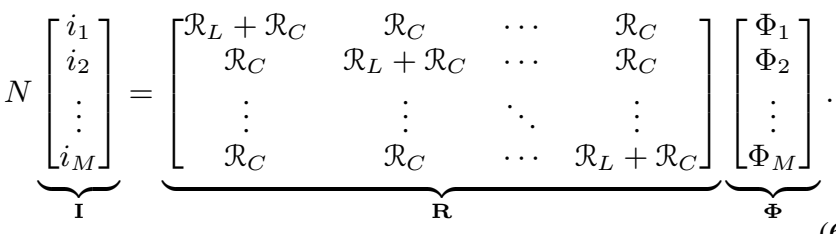

The equation which describes the gyrator-capacitor model and the inductance-dual model is the time derivative of (6):

$$
N \frac{d \mathbf{I}}{d t}=\mathbf{R} \frac{d \boldsymbol{\Phi}}{d t}=\mathcal{R}_{L} \frac{d \boldsymbol{\Phi}}{d t}+\mathcal{R}_{C} \frac{d \Phi_{C}}{d t}=\frac{\mathcal{R}_{L}}{N} \mathbf{V}+\frac{\mathcal{R}_{C}}{N} \sum_{x=1}^{M} v_{x}
$$

Note that $\mathbf{L}=N^{2} \mathbf{R}^{-1}$. As a result:

$$
\begin{gathered}
L_{S}=\frac{N^{2}\left(\mathcal{R}_{L}+(M-1) \mathcal{R}_{C}\right)}{\mathcal{R}_{L}\left(\mathcal{R}_{L}+M \mathcal{R}_{C}\right)}=L_{\mu}+L_{l}, \\
L_{M}=\frac{-N^{2} \mathcal{R}_{C}}{\mathcal{R}_{L}\left(\mathcal{R}_{L}+M \mathcal{R}_{C}\right)}=-\frac{1}{M-1} L_{\mu}, \\
L_{l}=\frac{N^{2}}{\mathcal{R}_{L}+M \mathcal{R}_{C}}=L_{S}+(M-1) L_{M}, \\
L_{\mu}=\frac{N^{2}(M-1) \mathcal{R}_{C}}{\mathcal{R}_{L}\left(\mathcal{R}_{L}+M \mathcal{R}_{C}\right)}=-(M-1) L_{M} .
\end{gathered}
$$

In a highly coupled structure, $\mathcal{R}_{L} \ll \mathcal{R}_{C}$, the relationships between $L_{S}, L_{M}, L_{\mu}, L_{l}, \mathcal{R}_{L}$ and $\mathcal{R}_{C}$ become:

$$
\begin{gathered}
L_{S} \approx \frac{(M-1) N^{2}}{M \mathcal{R}_{L}}, \\
L_{M} \approx \frac{-N^{2}}{M \mathcal{R}_{L}}, \\
L_{l} \approx \frac{N^{2}}{M \mathcal{R}_{C}}, \\
L_{\mu} \approx \frac{(M-1) N^{2}}{M \mathcal{R}_{L}} .
\end{gathered}
$$

To design a multiphase coupled inductor, it is important to be able to estimate the dc flux density in each portion of the core. In the inductance-dual model, the current that goes through $L_{C}=1 / \mathcal{R}_{C}, i_{C}$, is related to $\Phi_{C}$. The current in each of the legs, $i_{L 1}, i_{L 2}, \ldots, i_{L M}$ is related to the flux going through the corresponding outer leg of the core, $\Phi_{1}, \Phi_{2}, \ldots, \Phi_{M}$ :

$$
\begin{gathered}
\frac{d \Phi_{C}}{d t}=\frac{\sum_{x=1}^{M} v_{x}}{N}=\frac{1}{\mathcal{R}_{C}} \frac{d i_{C}}{d t}, \\
\frac{d \Phi_{x}}{d t}=\frac{v_{x}}{N}=\frac{1}{\mathcal{R}_{L}} \frac{d i_{L x}}{d t} .
\end{gathered}
$$


If the model in Fig. 13 is used, then

$$
\Phi_{C}=\frac{i_{C}}{\mathcal{R}_{C}} ; \Phi_{x}=\frac{i_{L x}}{\mathcal{R}_{L}} .
$$

Assuming the dc currents of all winding are all equal $I_{1}=$ $I_{2}=\ldots=I_{M}=I_{D C}$, and the output current $I_{o}=M I_{D C}$, the dc flux densities in the outer legs $\left(\Phi_{L, D C}\right)$ and center leg $\left(\Phi_{C, D C}\right)$ of the magnetic core, which determines the peak flux density when the system is working at full load, are functions of $M, N, \mathcal{R}_{L}, \mathcal{R}_{C}$, and $I_{D C}$ :

$$
\begin{gathered}
\Phi_{L, D C}=\frac{N I_{D C}}{\mathcal{R}_{L}+M \mathcal{R}_{C}}=\frac{N I_{o}}{M\left(\mathcal{R}_{L}+M \mathcal{R}_{C}\right)}=\frac{L_{l}}{M N} I_{o}, \\
\Phi_{C, D C}=\frac{M N I_{D C}}{\mathcal{R}_{L}+M \mathcal{R}_{C}}=\frac{N I_{o}}{\mathcal{R}_{L}+M \mathcal{R}_{C}}=\frac{L_{l}}{N} I_{o} .
\end{gathered}
$$

If current is balanced, the dc fluxes in the outer legs and center leg of the magnetic core are only determined by the leakage inductance $L_{l}$ and the output current $I_{o}$. The geometry of the core should be designed based on $L_{l}, I_{o}$, and current ripple, as discussed in more detail in the following section.

\section{Multiphase Coupled Inductor Buck Converter}

Coupled inductors can greatly enhance the performance of multiphase buck converters [1]-[16]. By coupling the multiple inductors with a single high-permeability magnetic core, one can significantly reduce the current ripple in each of the phase in order to reduce the conduction loss in switches, windings, and printed circuit board. One can, of course, achieve, the same ripple reduction in a single-phase or multiphase uncoupled design by using a larger inductor value, but this has two important disadvantages: It increases the energy storage, and thus increases the size and loss of the inductor. And it slows the transient response. Since these two effects scale together, we focus on considering ripple reduction for a fixed transient response impedance, and thus show the improvement available through coupling without the disadvantages of increasing inductance in the absence of coupling.

Even without coupling, the use of multiple phases can reduce ripple through partial cancellation at the output by multi-phase interleaving [52]-[55]. To compare an uncoupled multiphase converter to a single-phase converter, we apply the constraint of equal transient response. With $M$ phases, the reaction of the system to a perturbation in duty cycle across all phases, or to simply switching all phases high or low in a more urgent situation, is an equal perturbation applied to all $M$ inductors, effectively connected in parallel. Thus, for equal transient response, the parallel combination of $M$ inductors in the multiphase circuit should be equal to the value of the inductor used in the single-phase circuit: $L_{1 \phi}=L_{M \phi} / M$, where $L_{M \phi}$ is the per-phase uncoupled inductor value used in an $M$-phase circuit. With this choice, the absolute ripplecurrent amplitude in each phase is lower than the total ripple current in the single-phase converter by a factor of $1 / M$. The dc current in each phase is also lower than the overall $\mathrm{dc}$ current by the same factor, so each inductor of the $M$-phase design has the same current ripple ratio as the inductor with the same inductance in a single-phase design.

In the multi-phase converter, the total output capacitor current ripple amplitude, after partial cancellation of the ripple

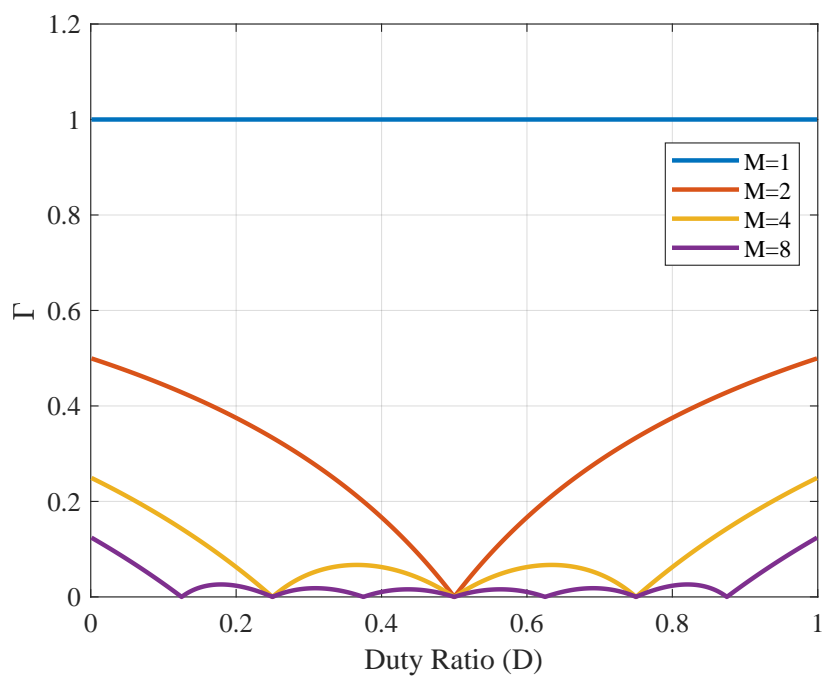

Fig. 23. Output current ripple reduction factor $(\Gamma)$ for an $M$-phase interleaved buck converter with duty ratio $D$. $\Gamma$ quantifies the benefit of interleaving for output current ripple reduction in multiphase buck converters, whether coupled or not. The maximum $\Gamma$ is $1 / M$. As $M$ increases, $\Gamma$ decreases.

current when the individual phase currents are combined, is reduced compared to the ripple in the single-phase converter by a factor that depends on the relationship between the duty ratio and the number of phases. As the way the phases interact depends on how many phases are energized simultaneously, it is convenient to introduce an integer index $k$ such that $\frac{k}{M} \leq D<\frac{k+1}{M}$. In terms of $k$, duty ratio $D$ and number of phases $M$, the output ripple of an interleaved uncoupled multiphase buck converter is reduced relative to that of a single-phase converter with the same response time by a factor

$$
\Gamma \stackrel{\text { def }}{=} \frac{\Delta i_{o}^{M \phi}}{\Delta i_{o}^{1 \phi}}=\frac{(k+1-D M)(D M-k)}{(1-D) D M^{2}},
$$

where we use the notation $\Delta i_{o}^{M \phi}$ to indicate peak-to-peak current ripple amplitude with the subscript $o$ indicating the output, or overall ripple after the phase currents are combined, and the superscript $M \phi$ indicates an $M$-phase converter. $\Gamma$ quantifies the benefits of interleaving for output current ripple reduction. It is similar to the factor $\delta$ in [55], except that $\Gamma$ is normalized by $M$ for equal transient response in the two converters being compared.

Fig. 23 plots $\Gamma$ as a function of $M$ and $D . \Gamma$ decreases as $M$ increases. When the duty cycle is near an integer multiple of $1 / M, \Gamma$ approaches 0 , indicating fully cancelled output current ripple. The advantage of coupling is that some of this benefit of output current ripple reduction can be extended to the ripple in each phase, and indeed, practical designs can approach the full reduction factor given by (21) with strong coupling.

We wish to consider coupled designs in the same framework of fixing transient response and varying other parameters, so we first evaluate the transient response. We can do this based on any of the models, considering either a small-signal perturbation to $D$ or the large signal response with all phases switch high or low, with equivalent results in all cases. For example, [10] examines a small-signal duty ratio perturbation using the inductance-matrix model. The appendix analyses the 
inductance-dual model with a small signal duty ratio perturbation. In all cases the result is that impedance of the whole structure to this common-mode excitation is the impedance of the parallel combination of all the leakage inductances, $L_{l} / M$. Perhaps the most intuitive way to understand this is to consider the transformer models (Figs. 15 and 16) with all of the $(+)$ terminals connected together and all of the () terminals connected together. In Fig. 15, the transformer equation requires all of the transformer winding voltages to sum to zero, and by symmetry, the only way for this to happen is with zero voltage across all of them, and thus zero voltage across the magnetizing inductances. The transformer portion of the model is transparent to this common mode excitation, and the impedance is just that of the leakage inductances. A similar argument applies to the model of Fig. 16.

Thus, the leakage inductance $L_{l}$ determines the transient impedance [57]. For ripple comparisons, we want to consider holding the leakage inductance fixed as we vary the coupling, equal to the per-phase inductance $L_{1 \phi}$ in the uncoupled case. The per-phase transient inductance (termed $L_{p t r}$ in [10]) is equal to $L_{l}$ and the overall transient inductance, $L_{o t r}=L_{l} / M$. As is shown in Table III, $L_{l}$ is given in terms of the parameters of other models as

$$
L_{l}=L_{S}+(M-1) L_{M}=\frac{N^{2}}{\mathcal{R}_{L}+M \mathcal{R}_{C}} .
$$

The steady-state ripple in the coupled case has been calculated in many different papers, with equivalent results, even though different formulations are used. Early papers such as [1] and [9] focus on two-phase converters with a limited range of duty cycles. In [2], this was generalized for an arbitrary number of phases, for duty ratio $D \leq 1 / M$. The analysis in [10] considers arbitrary duty ratios using the index $k$ as defined in conjunction with (21). The steady state phase ripple is described in [10] by an effective inductance ${ }^{1}$, the so-called "per-phase steady-state inductance," $L_{p s s}$. This is defined as an the inductance value that would, if used for discrete, uncoupled inductors in each phase of the same converter, result in the same peak-to-peak steady-state current ripple amplitude. It is given in terms of the inductance matrix parameters as

$$
L_{p s s}=\frac{(1-\alpha)(1+(M-1) \alpha)}{1+\left(M-2 k-2+\frac{k(k+1)}{M D}+\frac{M D(M-2 k-1)+k(k+1)}{M(1-D)}\right) \alpha},
$$

where $\alpha$ is the coupling factor $-L_{M} / L_{S}$ used in [9]. This can be used to find the ripple amplitude in each phase winding in the standard equation for ripple in a buck converter

$$
\Delta i_{p}=\frac{V_{O U T}(1-D) T}{L_{p s s}},
$$

where we again use the notation $\Delta i$ to indicate peak-to-peak ripple, and the subscript $p$ indicates the ripple in the winding of one of the $M$ phases. The superscript $M \phi$ is dropped because the subscript $p$ only applies when there are multiple phases.

We are now equipped to quantify the reduction in phase current ripple afforded by multiphase coupling, while keeping

\footnotetext{
${ }^{1}$ The approach in [10] uses a suite of four effective inductance parameters. These are reviewed in the appendix and derived based on the inductance-dual model, demonstrating the equivalence of the inductance-dual model to the inductance-matrix model used in [10].
}

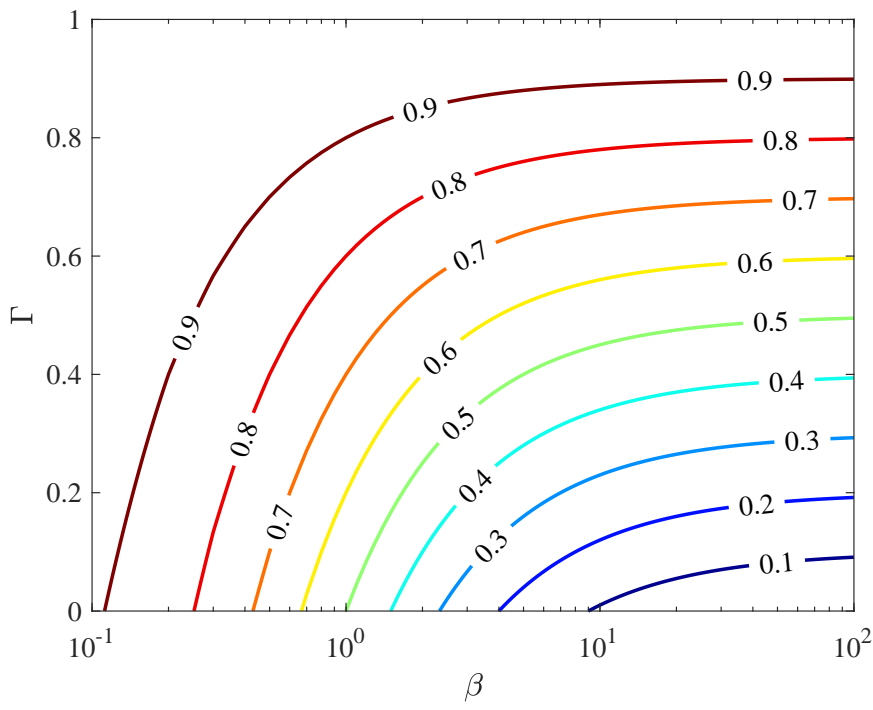

Fig. 24. Contours of phase current ripple reduction factor $(\gamma)$ for a multiphase coupled buck converter as a function of the output current ripple factor $(\Gamma)$ and coupling coefficient $(\beta)$. As $\beta$ increases, $\gamma$ approaches $\Gamma$, indicating that coupling extends the output current ripple improvement achieved through multiphase interleaving to the individual phase currents.

the transient response constant. We define a phase ripple current reduction ratio $\gamma$ as

$$
\gamma \stackrel{\text { def }}{=} \frac{\Delta i_{p}^{c p}}{\Delta i_{p}^{\text {noncp }}}
$$

with the inductance value in the uncoupled case equal to the leakage inductance of the coupled system for equal transient response. We use the superscripts $c p$ or noncp to indicate the coupled or uncoupled cases. Because (24) is unchanged for these two cases,

$$
\gamma=\frac{L_{l}}{L_{p s s}}
$$

To consider $\gamma$ or similar performance metrics as a function of the magnetic structure design, various papers in the literature use different parameters to describe the degree of coupling. The traditional parameter is the coupling factor $\alpha \stackrel{\text { def }}{=}-L_{M} / L_{S}$ used in [9]; for strong coupling, $\alpha$ approaches 1. In [1], the parameter $\rho \stackrel{\text { def }}{=} L_{\mu} / L_{l}$ is used. This allows a straightforward application to the case of fixed $L_{l}: \rho$ is simply the value of $L_{\mu}$ normalized to the leakage inductance. For the inductance-dual model, it is convenient to use a reluctance ratio $\beta \stackrel{\text { def }}{=} M \mathcal{R}_{C} / \mathcal{R}_{L}$ which is the ratio of center post reluctance to the parallel combination of all the outer leg reluctances. Note that $\rho$ is closely related to $\beta$ by $\rho=\frac{M-1}{M} \beta$. Conversions between different parameterizations are provided in Table III.

By combining (26) with calculations of its parameters based on the various models, we can write expressions for $\gamma$ as a function of the parameters $\alpha, \rho, \beta$ that are consistent with the equations corresponding equations in [9] and [1], for the scope of those equations (2-phase only and $D \leq 1 / M$, respectively). 

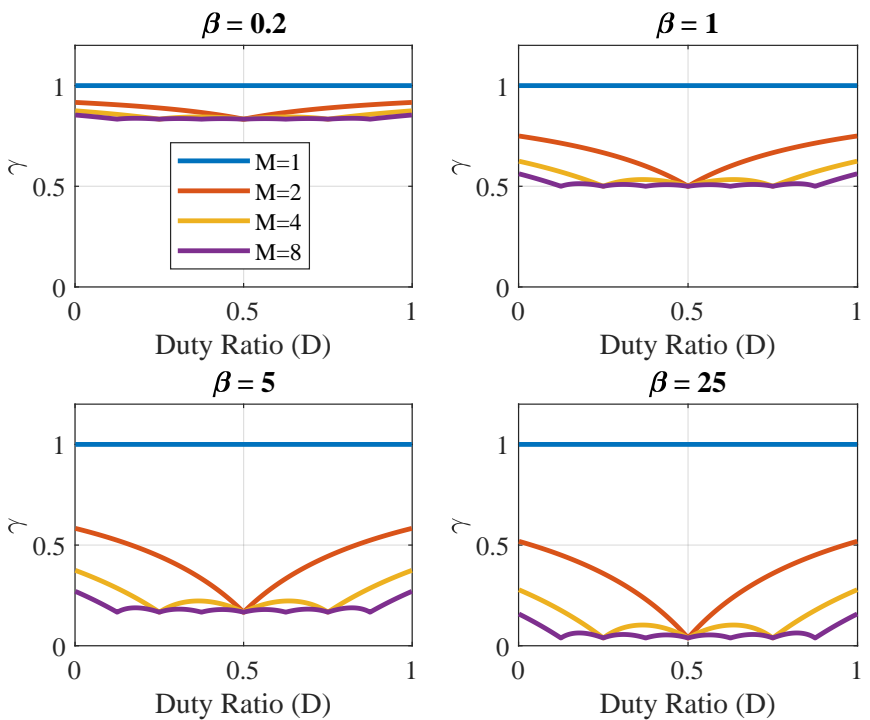

Fig. 25. Phase current ripple reduction factor $\gamma$ for a multiphase coupled buck inductor as a function of the duty ratio $(D)$ for various numbers of phases $(M)$ and reluctance ratios $\left(\beta=M \mathcal{R}_{C} / \mathcal{R}_{L}\right)$. A lower $\gamma$ indicates more winding current ripple reduction. A high $\beta$ indicates strong coupling, and a low $\beta$ indicates weak coupling.

Perhaps most elucidative is the expression in terms of coupling factor $\beta$ and output current ripple reduction factor $\Gamma$ :

$$
\gamma=\frac{\frac{1}{\beta}+\Gamma}{\frac{1}{\beta}+1}=\frac{1+\beta \cdot \Gamma}{1+\beta} .
$$

As illustrated in Fig. 24, this equation shows how the benefits of multiphase interleaving in reducing the output current ripple $(\Gamma)$ are extended to phase current ripple reduction by the coupling factor $(\beta)$. As coupling increases (a high value of $\beta$ ), the ripple reduction achieved in the phases approaches the ripple reduction in the combined output, $\Gamma$. This can be intuitively understood from the transformer model in Fig. 15: as coupling increases and $L_{\mu}$ becomes large, the transformer enforces equal current ripple in all phases, equal to the ripple that would be seen the output after they all combine. As the coupling decreases, the result strays from this ideal. For small $\Gamma$, such as at duty cycle near an integer multiple of $1 / M$, it approaches $\gamma=1 /(1+\beta)$. Fig. 25 plots $\gamma$ for a range of $D, M$, and $\beta . \gamma$ is always between zero and one, with a smaller $\gamma$ being better, indicating reduced ripple, or, if ripple is held fixed, an opportunity to reduce inductance to make the magnetic components smaller and speed up transient response. We can see that for sufficiently large $\beta$, the ripple reduction $\gamma$ approaches $\Gamma$. For moderate $\beta$, if $D$ is close to an integer multiple of $1 / M$, the ripple reduction approaches $1 /(1+\beta)$ as discussed above: for example, when $\beta=1$ the curves all go to $1 / 2$ at integer multiple of $1 / M$.

In practice, $\beta$ can be increased by reducing $\mathcal{R}_{L}$, by using high permeability core material, reducing length of the legs, and increasing area of the legs. $\mathcal{R}_{C}$ is then adjusted to maintain the selected $L_{l}$ to meet the transient requirements while maintain a small ripple. Trade-offs exist between core loss, saturation margin, energy storage requirements, and transient response. In an optimal design, the core loss, winding loss,
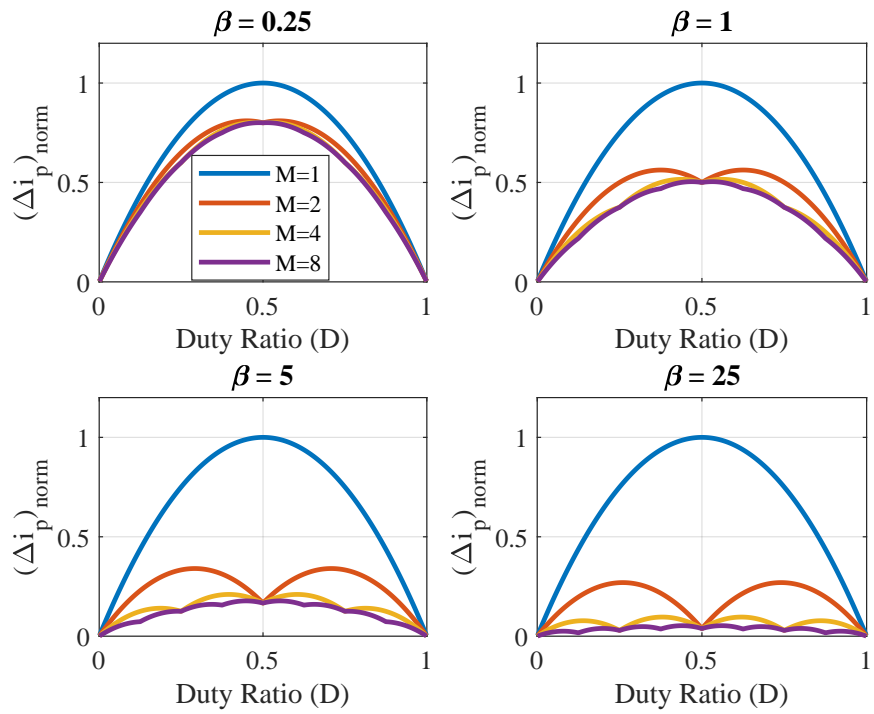

Fig. 26. Normalized winding current ripple $\left(\Delta i_{p}\right)_{\text {norm }}$ for a multiphase coupled buck inductor as a function of the duty ratio $(D)$ for various numbers of phases $(M)$ and reluctance ratios $\left(\beta=M \mathcal{R}_{C} / \mathcal{R}_{L}\right)$. A lower $\left(\Delta i_{p}\right)_{n o r m}$ indicates smaller absolute winding current ripple. A high $\beta$ indicates strong coupling, and a low $\beta$ indicates weak coupling.

TABLE II

Key Parameters for Multiphase Coupled Buck Converters

\begin{tabular}{c|c}
\hline Coupling Parameter & $\beta=\frac{M \mathcal{R}_{C}}{\mathcal{R}_{L}}=\frac{M}{M-1} \frac{L_{\mu}}{L_{l}}=\frac{-M \cdot L_{M}}{L_{S}+(M-1) L_{M}}$ \\
\hline Output Ripple Reduction & $\Gamma=\frac{\Delta i_{o}^{M \phi}}{\Delta i_{o}^{1 \phi}}=\frac{(k+1-D M)(D M-k)}{(1-D) D M^{2}}$ \\
\hline Phase Ripple Reduction & $\gamma=\frac{\Delta i_{p}^{c p}}{\Delta i_{p}^{n o p c p}}=\frac{1+\beta \Gamma}{1+\beta}$ \\
\hline Normalized Output Current & $\left(\Delta i_{p}\right)_{\text {norm }}=\frac{\Delta i_{c p, \text { phase }}}{\Delta i_{\text {max }, c p, p h a s e}}=4 D(1-D) \gamma$ \\
\hline
\end{tabular}

efficiency, power density, and transient and steady-state performance are highly correlated and need to be jointly optimized for a given design specification. For example, the optimization in [1] uses such optimizations to show how coupling can reduce volume and loss while holding transient response fixed, or improve transient response while holding volume and loss fixed. The number of phases is also considered, and for the parameters considered in that work, it is shown that 4 to 6 phases is preferred. With fewer phases, there is less of a benefit from coupling, whereas with more phases, general magnetics scaling trends [56] that lead to lower efficiency and power density with many small inductors kick in.

Short of a full optimization as in [1], a good strategy to design coupled inductors for multiphase buck converter is:

1) Selected a magnetic structure with $\mathcal{R}_{L} \ll \mathcal{R}_{C}$;

2) Choose an appropriate per-phase transient inductance $\left(L_{l}\right)$ based on the tradeoff between transient response and the overall output voltage ripple, assuming that a high $\beta$ will allow approaching a ripple reduction close the the ideal of $\gamma=\Gamma$.

3) Determine the required value of $\mathcal{R}_{L}+M \mathcal{R}_{C}$ based on the selected $L_{l}$ and $N$.

4) Design the magnetic structure (material and geometry) to minimize $\mathcal{R}_{L}$ and adjust $\mathcal{R}_{C}$ to maintain the selected 


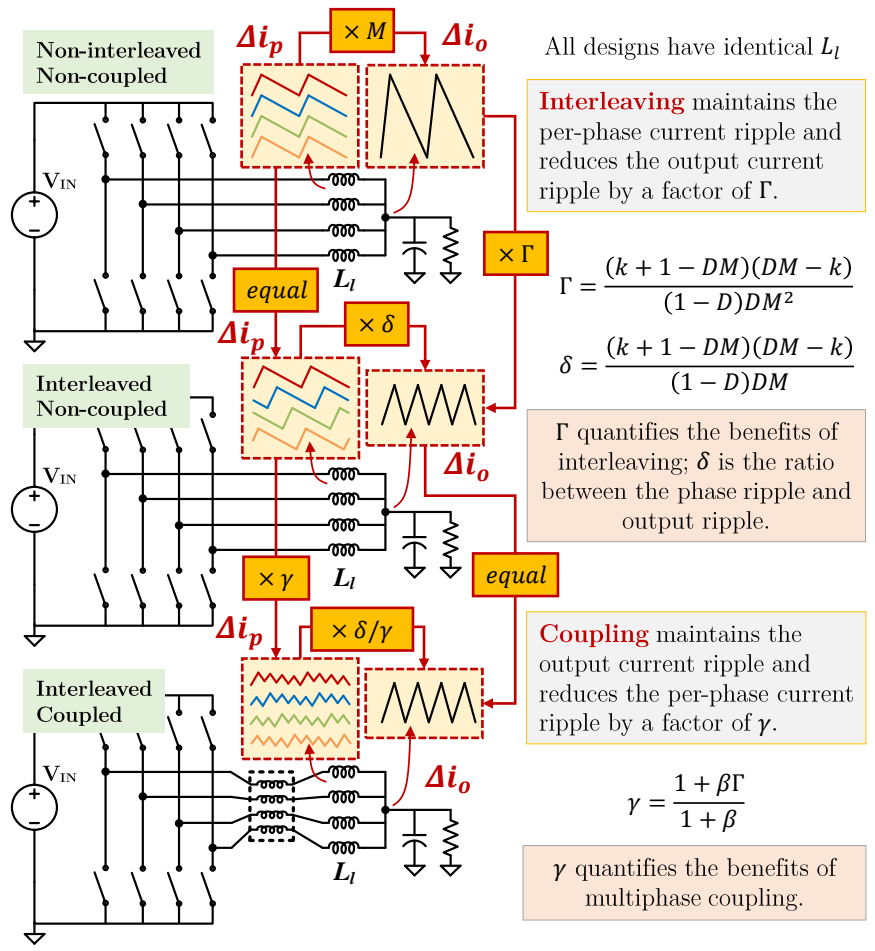

Fig. 27. Summary of the key design parameters of a few multiphase buck converters with the same transient performance. Interleaving maintains the per-phase current ripple and reduces the output current ripple by a factor of $\Gamma$. Coupling extends the benefits of interleaving to phase current reduction, and reduces the phase current ripple by a factor of $\gamma$.

$L_{l}$. In choosing the geometry parameters, cosiderations include minimizing the loss and ensuring enough margin to avoid saturation under balanced excitation.

5) Evaluate the flux under the expected worst-case mismatch between phase currents. If this leads to saturation, add small gaps in the outer legs as necessary to accommodate the mismatch.

There is always parasitic inductance adding to $L_{l}$ of the coupled inductor. When the targeted value of $L_{l}$ is small, the parasitic inductance outside the transformer may provide a significant fraction of the necessary leakage inductance, thus providing an opportunity to reduce the required inductance and thus reduce the energy storage required in the magnetic structure and reduce its size. In some cases, the parasitic inductance may exceed the targeted value of $L_{l}$, and careful layout to reduce parasitic inductance may be needed.

The absolute value of the current ripple per-phase impacts the loss in the windings and switches. If $D$ varies over a significant range in an application, it is useful to consider the worst-case absolute ripple for a given design, not just the ripple reduction ratio $\gamma$. The current ripple of each phase is

$$
\Delta i_{p}=\frac{V_{O U T}(1-D) T}{L_{p s s}}=\gamma \frac{V_{I N} D(1-D) T}{L_{l}} .
$$

A helpful way to consider this is to normalize it to the worstcase phase current ripple at $D=0.5$ with no coupling,

$$
\Delta i_{p}^{\max }=\frac{V_{O U T} T}{2 L_{l}}=\frac{V_{I N} T}{4 L_{l}},
$$

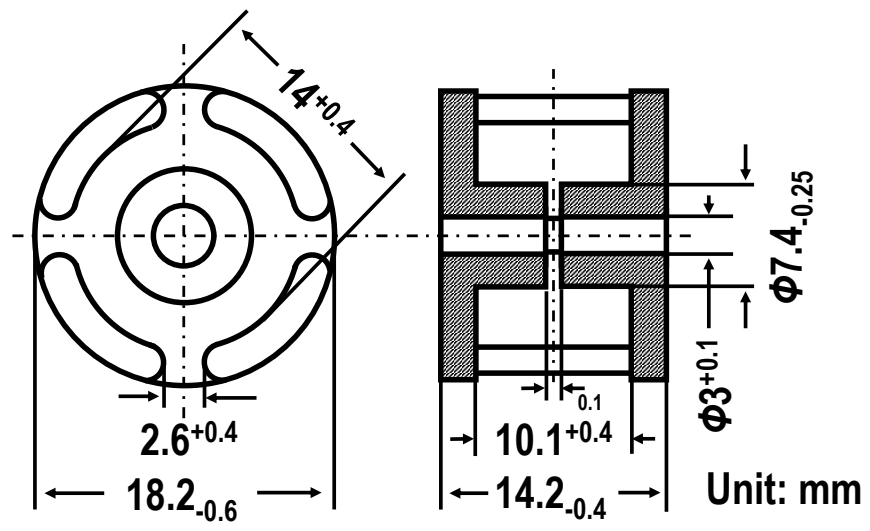

Fig. 28. The geometry of a four-phase coupled inductor with four outer legs and one center leg (dimension labeled in $\mathrm{mm}$ ). The effective length of each outer leg is $18 \mathrm{~mm}$. The effective area of each outer leg is $11.25 \mathrm{~mm}^{2}$. The effective area of the center leg is $45 \mathrm{~mm}^{2}$. The relative permeability of the core is 2300 . There is a $0.1 \mathrm{~mm}$ air gap in the center leg.

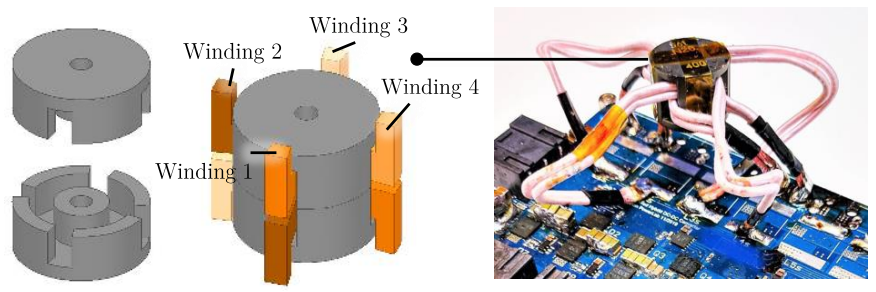

Fig. 29. An example four-phase coupled inductor buck converter. Each phase has a single turn. The extra winding outside the core (for current measurement) adds about $30 \mathrm{nH}$ parasitic inductance to each winding of the coupled inductor.

resulting in the normalized current ripple:

$$
\left(\Delta i_{p}\right)_{n o r m} \stackrel{\text { def }}{=} \frac{\Delta i_{p}}{\Delta i_{p}^{\text {max }}}=\gamma 4 D(1-D)=\frac{1+\beta \Gamma}{1+\beta} 4 D(1-D) .
$$

Fig. 26 plots this normalized current ripple across a range of $D, M$, and $\beta$. Both Figs. 26 and 25 illustrate the advantages of increasing the coupling and of increasing the number of phases. Fig. 25 directly shows how much benefit is provided at a given operating point, whereas Fig. 26 is useful for considering a range of operating points with different duty ratios, and assessing the ripple and ripple reduction at the worst-case point over that range.

Table II lists the key design parameters for multiphase coupled inductors. Fig. 27 illustrates the relationships among these parameters. Table III summarizes the equations for calculating various parameters based on the inductance-matrix model, inductance-dual model, and multiwinding transformer model $^{2}$.

\section{MOdel VerificATION}

A four-phase coupled inductor design was selected to verify the models discussed in this paper. This design is not optimized for a particular application. Fig. 28-29 show the geometry of a SIEMENS P1814 B65561-A0400 core with N26 material. The structure has four outer legs and one center leg.

\footnotetext{
${ }^{2}$ A software tool - Princeton CoupL - was developed based on Table II and is available at: http://www.princeton.edu/ minjie/coupL/coupL.html.
} 
TABLE III

Unified Model Parameter Equations for Multiphase Coupled Buck Inductors

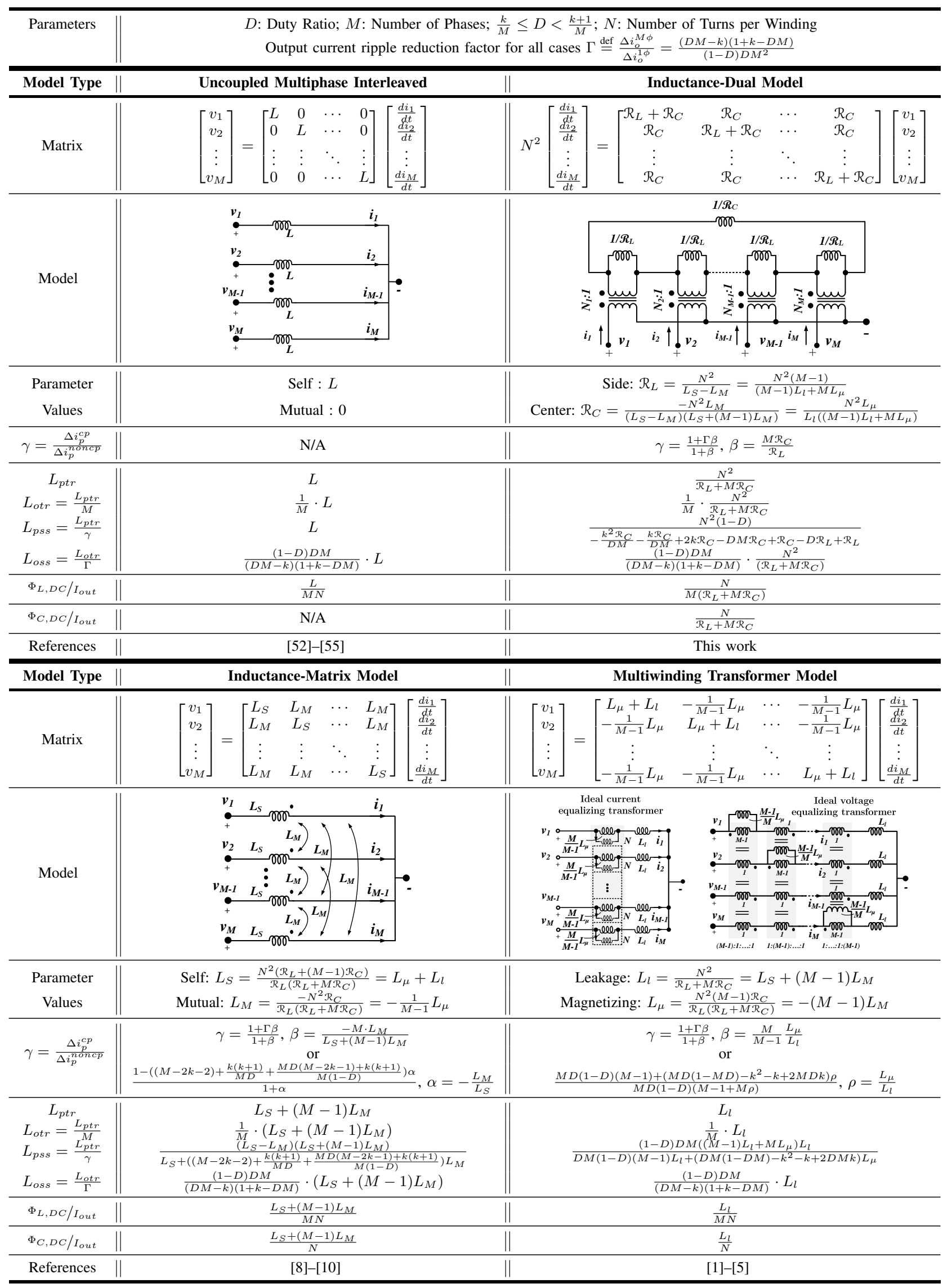



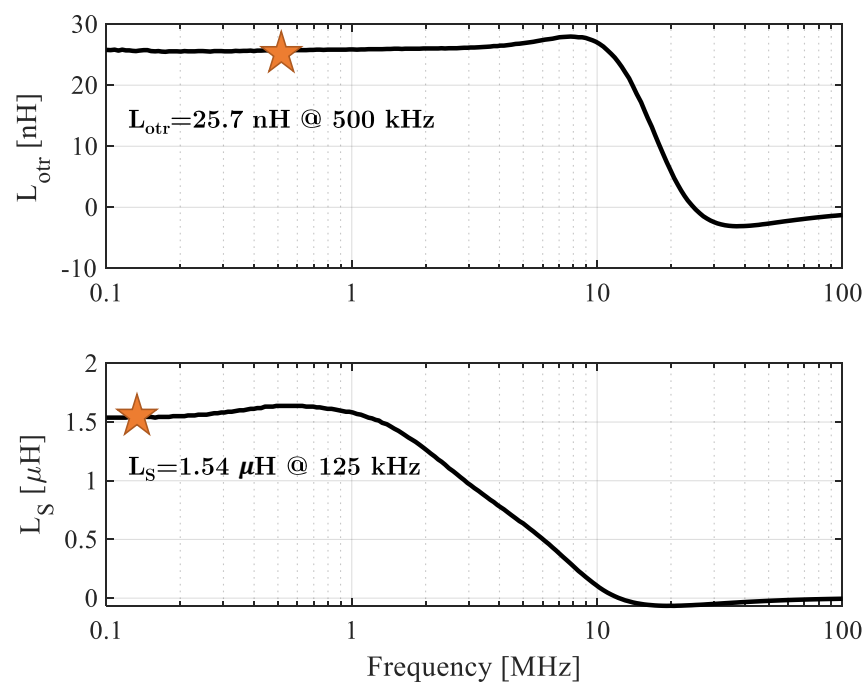

Fig. 30. Measurements used for parameter extraction from the fabricated coupled inductor. $L_{o t r}$ : measured inductance of four windings connected in parallel. $L_{S}$ : measured inductance of a single winding on an outer leg.

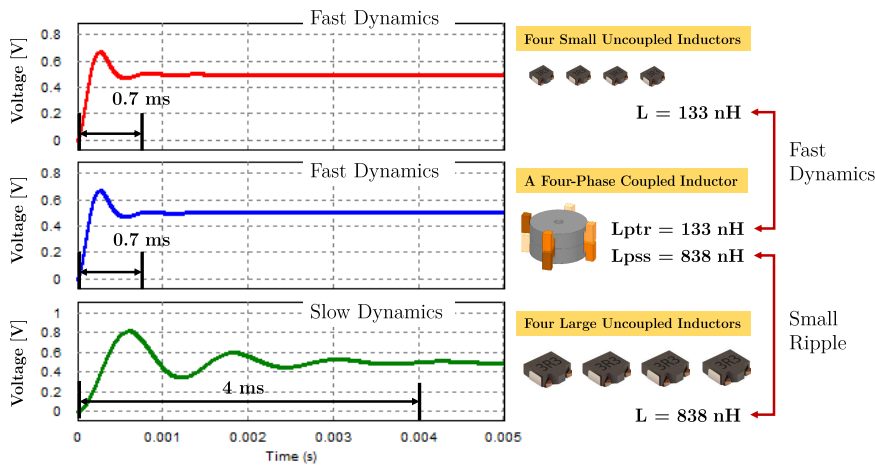

Fig. 31. Simulated transient output voltage waveforms of a four-phase buck converter with a) four uncoupled $133 \mathrm{nH}$ inductors; b) one four-phase coupled inductor with a per-phase transient inductance of $133 \mathrm{nH}$, and per-phase steady-state inductance of $838 \mathrm{nH}$; and c) four uncoupled $838 \mathrm{nH}$ inductors.

The reluctances $\mathcal{R}_{L}$ and $\mathcal{R}_{L}$ were calculated based on simple approximations. These values, denoted as $\mathcal{R}_{L}^{*}$ and $\mathcal{R}_{C}^{*}$, are $492,070 \mathrm{H}^{-1}$ and $1,860,700 \mathrm{H}^{-1}$, respectively. Fig. 30 shows the measured inductance of the prototype used for parameter extraction. With $N=1$, the measured inductance with four windings connected in parallel $L_{o t r}$ was $25.7 \mathrm{nH}$. The measured self inductance of a single winding with the others open circuited $L_{S}$ was $1.54 \mu \mathrm{H}$. As explained in Appendix II, these can be used was used to obtain values of $\mathcal{R}_{L}$ and $\mathcal{R}_{C}$ as

$$
\begin{gathered}
\mathcal{R}_{L}=\frac{N^{2}(M-1)}{M\left(L_{S}-L_{o t r}\right)}, \\
\mathcal{R}_{C}=\frac{N^{2}\left(L_{S}-M L_{o t r}\right)}{M^{2} L_{o t r}\left(L_{S}-L_{o t r}\right)} .
\end{gathered}
$$

resulting in $\mathcal{R}_{L}=496,100 \mathrm{H}^{-1}$ and $\mathcal{R}_{C}=2,307,600 \mathrm{H}^{-1}$. The extracted parameters match well with the calculated ones.

In the test circuit, the structure is connected with longer leads to facilitate current measurements. These leads add about $30 \mathrm{nH}$ of parasitic inductance in series with each winding. We can construct a model that includes this additional inductance
TABLE IV

Model Parameters For the PROTOTyPe Coupled InduCtor

\begin{tabular}{cc|c|c|c}
\hline Parameter & Symbol & Value for structure only & Value including $L_{p}$ \\
\hline Measured single winding & $L_{S}$ & $1.54 \mu \mathrm{H}$ & N/A \\
Measured shorted windings & $L_{o t r}$ & $25.7 \mathrm{nH}$ & N/A \\
Measured Parasitic Inductance & $L_{p}$ & N/A & N/A nH in each phase \\
\hline Extracted leg reluctance & $\mathcal{R}_{L}$ & $496,100 \mathrm{H}^{-1}$ & N/A \\
Extracted centerpost reluctance & $\mathcal{R}_{C}$ & $2,307,600 \mathrm{H}^{-1}$ & N/A \\
Calculated leg reluctance & $\mathcal{R}_{L}^{*}$ & $492,070 \mathrm{H}^{-1}$ & N/A \\
Calculated centerpost reluctance & $\mathcal{R}_{C}^{*}$ & $1,860,700 \mathrm{H}^{-1}$ & $2.04 \mu \mathrm{H}$ \\
\hline Inductance-dual & $\mid L_{L}$ & $2.01 \mu \mathrm{H}$ & $569 \mathrm{nH}$ \\
model & $L_{C}$ & $434 \mathrm{nH}$ & $133 \mathrm{nH}$ \\
model & $L_{l}$ & $103 \mathrm{nH}$ & $1.43 \mu \mathrm{H}$ \\
\hline Multiwinding transformer & $L_{\mu}$ & $1.43 \mu \mathrm{H}$ & $1.56 \mu \mathrm{H}$ \\
Inductance matrix & $L_{S}$ & $1.53 \mu \mathrm{H}$ & $-477 \mathrm{nH}$ \\
model & $L_{M}$ & $-477 \mathrm{nH}$ & $333 \mathrm{nH}$ \\
Effective inductance & $L_{\text {oss }}$ & $258 \mathrm{nH}$ & $838 \mathrm{nH}$ \\
parameters (Appendix I.) & $L_{p s s}$ & $705 \mathrm{nH}$ & $33.2 \mathrm{nH}$ \\
for $D=1 / 6$ & $L_{o t r}$ & $25.8 \mathrm{nH}$ & $133 \mathrm{nH}$ \\
\hline Key design parameters & $L_{p t r}$ & $103 \mathrm{nH}$ & 14.3 \\
for $D=1 / 6$ & $\beta$ & 18.5 & $10.0 \%$ \\
& $\Gamma$ & $10.0 \%$ & $15.8 \%$ \\
\hline & $\gamma$ & $14.6 \%$ & \\
\hline
\end{tabular}

as part of the same model structure, as shown in the second column of Table IV, or we can analyze or simulate the circuit using the model of the structure itself with separate inductances representing the parasitic lead inductance. The parameters for this approach are shown in the first column in Table IV. We neglect any mutual coupling between the parasitic inductances. Although the need for modeling the parasitic inductance in our case was driven by the use of extra instrumentation for demonstration purposes, it is important in practical applications as well, such as in fast-response converters where the leakage inductance of the structure is designed to be very low and so even small parasitic inductance becomes significant.

Fig. 31 shows the simulated open-loop startup voltage transient waveforms of three experimental designs. Design1 uses four small $133 \mathrm{nH}$ uncoupled inductors. Design-2 uses the four-phase coupled inductor which, with the parasitic inductance included in the model, is expected to have the same transient performance but with coupling reducing the ripple to the equivalent of that expected with $838 \mathrm{nH}$ per-phase inductance. Design-3 uses four big uncoupled $838 \mathrm{nH}$ inductors. The simulated output voltage transient waveforms of Design-1 and Design-2 are exactly the same. The output voltage transient of Design-3 has large overshoot and oscillation.

The inductance-dual model, multiwinding transformer model, and inductance-matrix model of the prototype coupled inductor are shown in Figs. 32-34, respectively. For clarity, we show these with the parasitic inductance modeled separately, although in practice, incorporating the parasitic inductance into the model makes modeling and analysis easier. In the multiwinding transformer model, $L_{l}$ is $103 \mathrm{nH}$ and $L_{\mu}$ is $1.43 \mu \mathrm{H}$. The input voltage is $3 \mathrm{~V}$, the average output current is $10 \mathrm{~A}$, the output capacitance is $200 \mathrm{mF}$, the output resistance is $0.05 \Omega$, the switching frequency is $125 \mathrm{kHz}$, and the duty ratio $D=1 / 6$. An un-coupled four-phase buck converter would achieve the same transient response with $133 \mathrm{nH}$ inductors in each phase. This results in per-phase current ripple of $25.1 \mathrm{~A}$ and rms current of $9.59 \mathrm{~A}$. With the prototype four-phase 


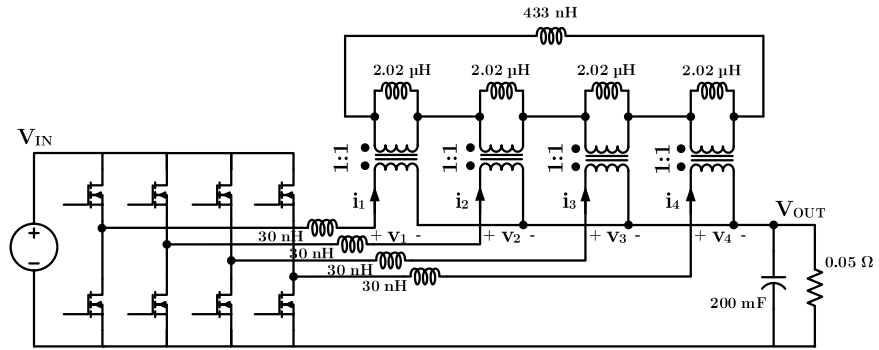

Fig. 32. SPICE simulation of the four-phase coupled inductor buck converter based on the inductance-dual model.

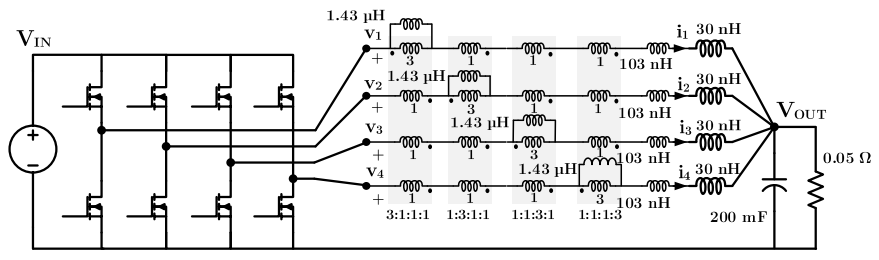

Fig. 33. SPICE simulation of the four-phase coupled inductor buck converter based on the multiwinding transformer model implemented with an ideal voltage equalization transformer.

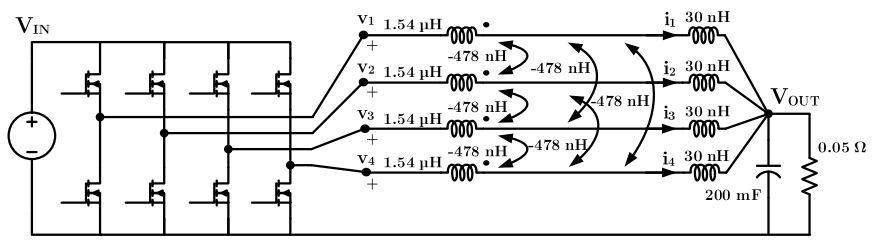

Fig. 34. SPICE simulation of the four-phase coupled inductor buck converter based on the inductance-matrix model.

coupled inductor, the transient response is the same, but the steady-state phase current ripple is reduced by coupling, to an expected value of $3.98 \mathrm{~A}, 15.8 \%$ of that of the uncoupled case. The expected rms current is $2.70 \mathrm{~A}, 28.1 \%$ of that of the uncoupled case.

Fig. 35 shows the simulated steady-state per-phase current of the three converters. Simulations of the coupled case were performed with the three different models in Figs. 32-34, with identical results. The per-phase current ripple of the converter with four small inductors was $25.1 \mathrm{~A}$, and the perphase current ripple of the buck converter with the coupled inductor was $3.98 \mathrm{~A}$. The simulation results match with the calculated results. Fig. 36 shows the measured waveforms of the experimental prototype. As expected, the measured perphase peak-to-peak current ripple is about $4 \mathrm{~A}$.

To demonstrate the capability of the inductance-dual model to include magnetic saturation, the design was modified by changing the number of turns on one of the windings to two, while leaving the others windings with one turn. This results in an asymmetric structure that saturates early with equal dc currents in the different legs. Fig. 37 shows a circuit simulation model (implemented in Powersim) with saturable inductor models. In this case, it was important to model the parasitic inductance separately from the coupled structure, such that the elements of the model correspond directly to the parts of the magnetic core. A non-linear current-flux-linkage relationship was implemented in the saturable inductor model in circuit

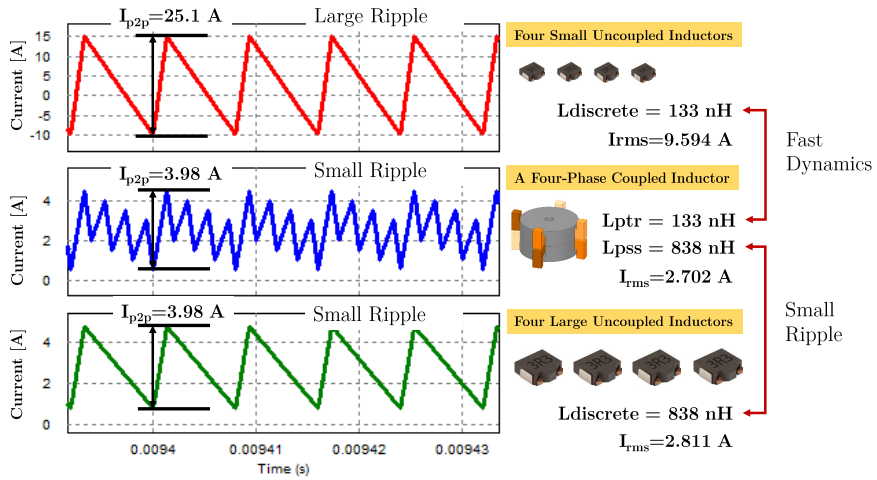

Fig. 35. Simulated steady-state winding current of a four-phase buck converter with a) four uncoupled $133 \mathrm{nH}$ inductors; b) one four-phase coupled inductor with a per-phase transient inductance of $133 \mathrm{nH}$, and per-phase steady-state inductance of $838 \mathrm{nH}$; and c) four uncoupled $838 \mathrm{nH}$ inductors.

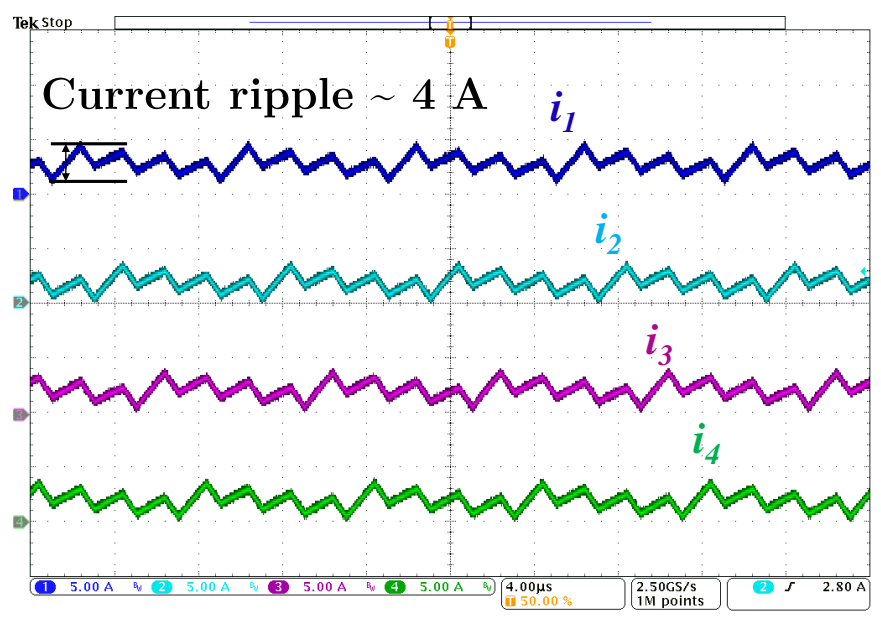

Fig. 36. Measured waveforms of the symmetric four-phase coupled inductor.

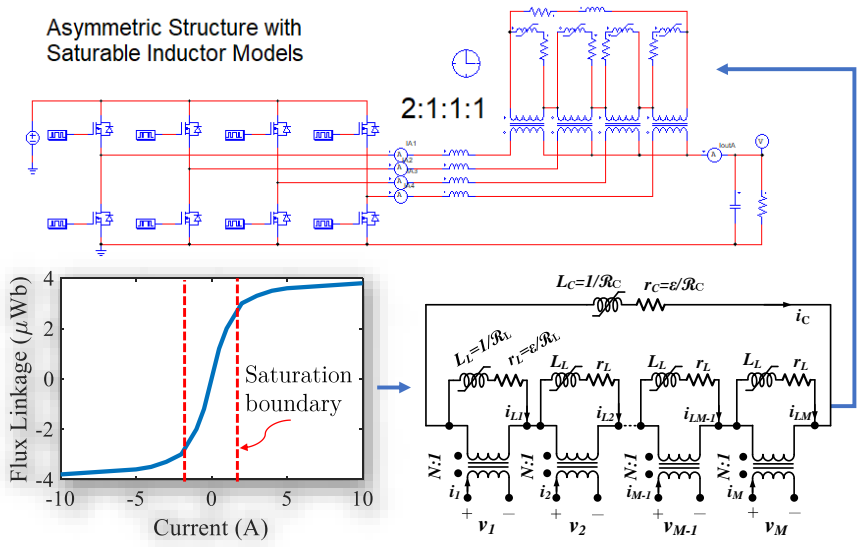

Fig. 37. Simulation setup in Powersim with asymmetric structure and saturable inductor models. The saturable inductor model represents non-linear current-flux-linkage relationships in circuit simulations.

simulations, and small resistors were used in series with each resistor to stabilize dc flux levels as per (2). The unbalanced MMF leads to unbalanced current and flux distribution, and may cause saturation when the flux mismatch is significant. Fig. 38 shows the measured waveforms of the modified design with $10 \mathrm{~A}$ as the overall output current. Strong asymmetry 


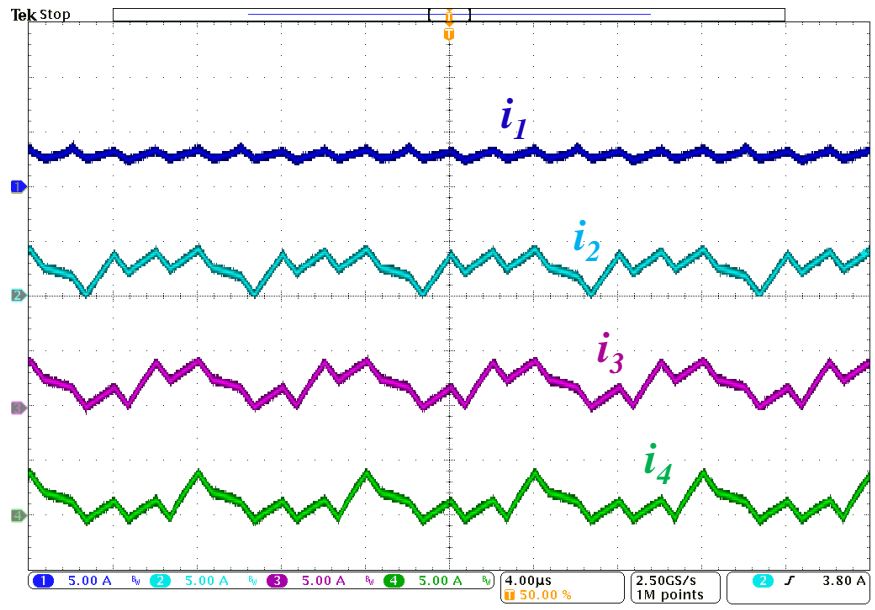

Fig. 38. Measured waveforms of the asymmetric four-phase coupled inductor. Winding \#1 has two turns, and Windings \#2-\#4 have a single turn.

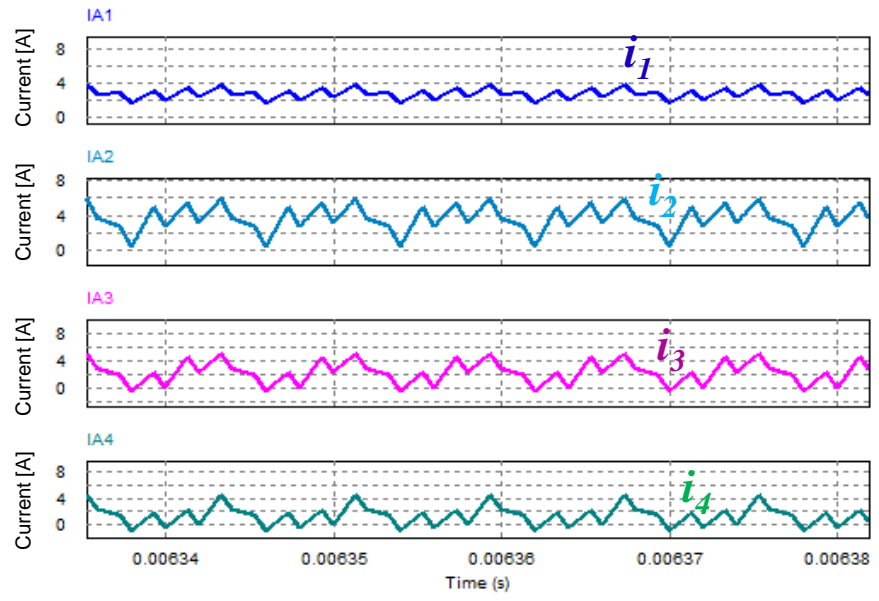

Fig. 39. Simulated waveforms of the asymmetric four-phase coupled inductor. Winding \#1 has two turns, and Windings \#2-\#4 have a single turn.

was observed in phase current, as predicted by the simulation results in Fig. 39.

The saturation flux density $B_{\text {sat }}$ of the SIEMENS N26 material is about $390 \mathrm{mT}$ at $25{ }^{\circ} \mathrm{C}$. The saturation flux $\Phi_{\text {sat }}=$ $B_{\text {sat }} A_{C}$ of the outer leg was $4.388 \mu \mathrm{Wb}\left(A_{C}=11.25 \mathrm{~mm}^{2}\right)$. $\mathcal{R}_{L}$ was $496,100 \mathrm{H}^{-1}$. The threshold current for the saturable inductor is about $2.17 \mathrm{~A}$. Fig. 40 shows the measured current waveforms when winding \#1 with two turns is saturated $\left(i_{1}\right)$. The total output current is about $20 \mathrm{~A}$. The average current per phase is about $5 \mathrm{~A}$. The per-phase inductance of this winding drops significantly as the inductor current $\left(i_{1}\right)$ approaches the saturation limit $(5 \mathrm{~A})$, which matches well with the simulated results in Fig. 41. Fig. 42 shows the simulated current of $L_{L}$ and $L_{C}$ which reflects the outer and center leg flux $\left(i_{L}=\Phi_{L} \mathcal{R}_{L}\right)$. The magnetic core was saturated when $i_{L}$ approaches $2.17 \mathrm{~A}\left(\Phi_{\text {sat }} \mathcal{R}_{L}\right)$. The non-linear saturation effects are captured in SPICE simulations.

\section{CONCLUSIONS}

This paper unifies the lumped circuit models for multiphase coupled inductors. Models including inductance-matrix

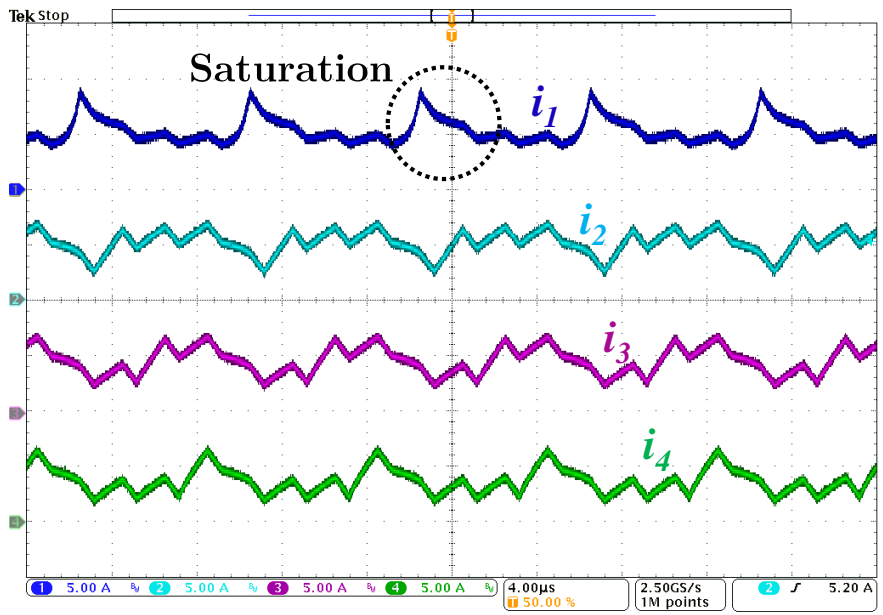

Fig. 40. Measured waveforms of the asymmetric coupled inductor with 2:1:1:1 turns ratio. The leg with two turns is saturated and a current spike is observed. The waveform patterns of the other three legs remain the same.

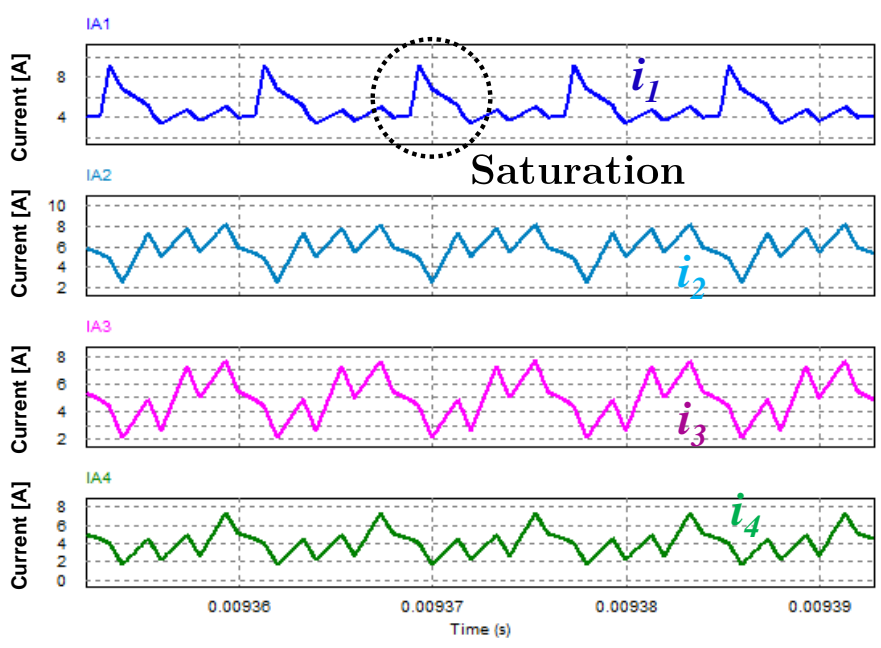

Fig. 41. Simulated waveforms of the asymmetric coupled inductor with 2:1:1:1 turns ratio.The leg with two turns is saturated and a current spike is observed. The waveform patterns of the other three legs remain the same.

models, multiwinding transformer models, extended cantilever models, magnetic-circuit models, gyrator-capacitor models, and inductance-dual models are summarized and compared. These models represent identical mathematical relationships in the multiphase coupled inductors, but reveal different physical fundamentals and distinct design insights. Inductance-matrix models, multiwinding transformer models, and extended cantilever models are math-based models. Magnetic-circuit models, gyrator-capacitor models, and inductance-dual models are physics-based models. All are equivalent in the linear case, and we provide formulas to convert parameters between the different models. The inductance-dual model is particularly useful amongst these models because it is directly built on elements in the magnetic-circuit model and offers convenience in circuit simulations. Core loss and saturation effects in each portion of the core can be captured.

It is shown that phase-current ripple reduction that coupling provides in a multiphase buck converter can be calculated with a simple equation in terms two parameters, $\Gamma$ which quantifies 


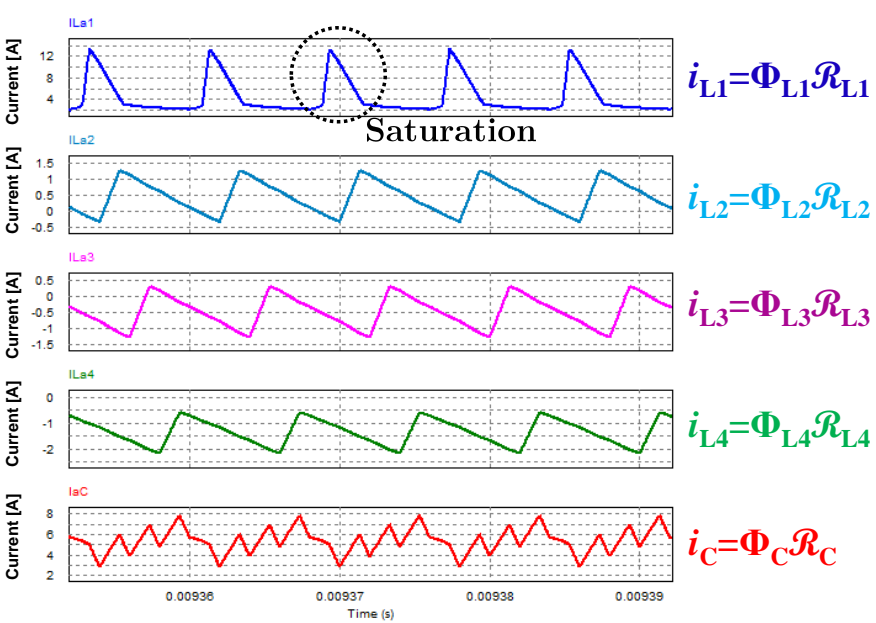

Fig. 42. Simulated current waveforms of $L_{L}$ and $L_{C}$ in the inductance-dual model when the inductors $L_{L}$ are implemented as saturable inductors. The leg with two turns is saturated. The inductance-dual model allows the magnetic flux be visualized as current, and enable rapid evaluation of the flux density.

the output ripple current reduction provided by multiphase interleaving, and $\beta$ which quantifies the degree of coupling. This formulation simplifies design and analysis calculations, while also providing insight on origin of the coupling benefit.

The models and design equations have been verified through theoretical derivation, SPICE simulations, and experimental measurements.

\section{ACKNOWLEDGEMENTS}

This work was supported by the the National Science Foundation under Award \#1847365. The authors would like to thank Dr. Yenan Chen of Princeton University for collecting the experimental data of this work during COVID-19.

\section{Appendix I: EfFective Inductance Performance PARAMETERS}

Four design parameters were defined in [10] based on the inductance-matrix model for a $M$-phase coupled inductor buck converter with an arbitrary duty ratio $D$ by defining an index $k$ to indicate the number of phases that are simultaneously energized. $D$ and $k$ are related by $\frac{k}{M} \leq D<\frac{k+1}{M}$ :

1) Overall steady-state inductance $\left(L_{o s s}\right)$ : the multiphase coupled inductor results in the same total output peak-topeak ripple current amplitude as a single discrete inductor with inductance $L_{\text {oss }}$.

2) Per-phase steady-state inductance $\left(L_{p s s}\right)$ : each phase of the multiphase coupled inductor has the same peak-topeak steady-state current ripple as with individual discrete inductors with inductance $L_{p s s}$;

3) Overall transient inductance $\left(L_{o t r}\right)$ : the multiphase coupled inductor behaves as a single discrete inductor with $L_{o t r}$ as the inductance for the purposes of evaluating the overall transient performance and small signal model;

4) Per-phase transient inductance $\left(L_{p t r}\right)$ : the same physical meaning as the overall transient inductance, but normalized on a per-phase basis by dividing the overall transient inductance by the number of phases.

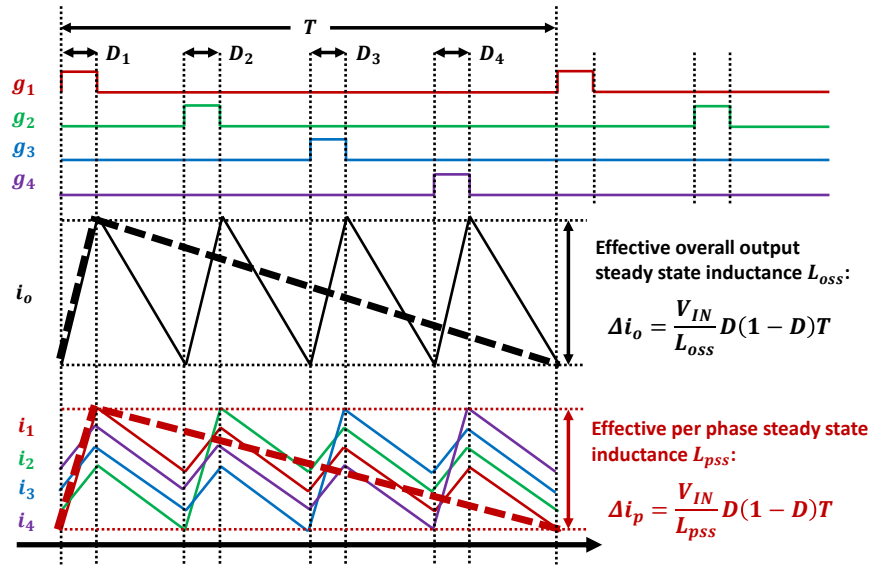

Fig. 43. Effective overall steady-state inductance $\left(L_{o s s}\right)$ and effective perphase steady-state inductance $\left(L_{p s s}\right)$ of the multiphase coupled inductor.

These parameters are expressed as functions of $L_{S}, L_{M}$, $D, M$, and $k$ in [10] based on the inductance-matrix model:

$$
\begin{aligned}
L_{o s s} & =\frac{(1-D) D M\left(L_{S}+L_{M}(M-1)\right)}{(D M-k)(1+k-D M)} \\
L_{p s s} & =\frac{\left(L_{S}-L_{M}\right)\left(L_{S}+(M-1) L_{M}\right)}{L_{S}+\left((M-2 k-2)+\frac{k(k+1)}{M D}+\frac{M D(M-2 k-1)+k(k+1)}{M(1-D)}\right) L_{M}} \\
L_{\text {otr }} & =\frac{L_{S}+(M-1) L_{M}}{M} \\
L_{p t r} & =L_{S}+(M-1) L_{M}
\end{aligned}
$$

With these design parameters, the steady-state output current ripple, the output small-signal model, per-phase current ripple, and per-phase small-signal model of the multiphase coupled inductor, working in continuous-conduction-mode, can be rapidly estimated by applying them in the standard equations for a single-phase buck converter with the same duty ratio $D$ and the same switching frequency $f$. Note that $L_{o s s}, L_{p s s}$, $L_{o t r}$ and $L_{p t r}$ are related by $\Gamma, \gamma$ and $M: L_{o t r} / L_{o s s}=\Gamma$, $L_{p t r} / L_{p s s}=\gamma, L_{p t r} / L_{o t r}=M$ and $L_{p t r}=L_{l}$.

These effective inductance values are also derived below based on the inductance-dual model, for reference and to demonstrate the equivalance of the models.

\section{A. Effective Overall Steady-State Inductance ( $\left.L_{\text {oss }}\right)$}

The effective overall steady-state inductance can be used to predict the effective output current ripple of the multiphase buck converter as if the multiphase coupled inductor is one single discrete inductor (Fig. 43). In a $M$ phase coupled inductor buck converter with $V_{I N}$ as the input voltage, an arbitrary duty ratio $\frac{k}{M} \leq D<\frac{k+1}{M}$, and $V_{O U T}=D V_{I N}$ as the output voltage, the derivative of the overall output current is the summation of the derivative of the current of all phases:

$$
\frac{d i_{o}}{d t}=\frac{d i_{1}}{d t}+\frac{d i_{2}}{d t}+\ldots+\frac{d i_{M}}{d t} .
$$

- During $\frac{k}{M} T<t<D T$, the overall current ramps up. There are always $k+1$ inductors which have $V_{I N}-V_{O U T}$ across them, and $M-k-1$ inductors which have $-V_{O U T}$ 
across them for an on period $\left(D-\frac{k}{M}\right) T$. The $\{k+1\}^{t h}$ inductor has $V_{I N}-V_{O U T}$ across it.

- During $D T<t<\frac{k+1}{M} T$, the overall current ramps down. There are always $i$ inductors which have $V_{I N}-V_{O U T}$ across them, and $M-k$ inductors which have $-V_{O U T}$ across them for an off period $\left(\frac{k+1}{M}-D\right) T$. The $\{k+1\}^{\text {th }}$ inductor has $-V_{O U T}$ across it.

Substituting Eq. (34) into Eq. (7), we find that the current ripple during the ramp up and ramp down period are:

$$
\begin{gathered}
\Delta i_{o}^{u p}=\frac{\left(\mathcal{R}_{L}+M \mathcal{R}_{C}\right)(k+1-D M)(D M-k)}{D M N^{2}} V_{O U T} T, \\
\Delta i_{o}^{\text {down }}=-\frac{\left(\mathcal{R}_{L}+M \mathcal{R}_{C}\right)(k+1-D M)(D M-k)}{D M N^{2}} V_{O U T} T .
\end{gathered}
$$

Note that in steady-state, $\Delta i_{o}^{s s}=\Delta i_{o}^{u p}=-\Delta i_{o}^{\text {down }}=$ $\frac{V_{O U T}}{L_{s s}}(1-D) T$. A single phase inductor with the same ripple would have a value

$$
L_{\text {oss }}=\underbrace{\frac{N^{2}}{\mathcal{R}_{L}+M \mathcal{R}_{C}}}_{L_{p t r}} \times \underbrace{\frac{(1-D) D M}{(k+1-D M)(D M-k)}}_{1 / \delta} .
$$

for an $M$ phase coupled buck converter with $\frac{k}{M} \leq D<\frac{k+1}{M}$. Here $L_{p t r}=\frac{N^{2}}{\mathcal{R}_{L}+M \mathcal{R}_{C}}$ is the equivalent inductance of the side leg of the coupled inductor as if the center leg is evenly divided into $M$ pieces. It is also the per-phase transient inductance as will be derived. Appendix II introduced a method to measure $L_{p t r} . \delta=\frac{(k+1-D M)(D M-k)}{(1-D) D M}$ is the normalized ripple amplitude of an uncoupled interleaved $M$-phase buck converter with duty ratio $D$ (Fig. 23) [52]-[55].

For an $M$-phase coupled inductor buck converter operating in continous conduction mode with duty ratio $D$, switching period $T$, and output voltage $V_{O U T}$, the peak-to-peak ripple of the overall output current is a simple function of $L_{o s s}$ :

$$
\Delta i_{o}=\frac{V_{O U T}(1-D) T}{L_{o s s}} .
$$

\section{B. Effective Per-Phase Steady-State Inductance $\left(L_{p s s}\right)$}

If $\frac{k}{M} \leq D<\frac{k+1}{M}$, during the $0<t<D T$ period, for each phase, there are $k+1$ numbers of $\left(D-\frac{k}{M}\right) T$ sub-periods, in which $k+1$ phases have $V_{I N}-V_{O U T}$ across them, and $M-k-1$ phases have $-V_{O U T}$ across them. The per-phase current ramps up. The current ripple in each winding during the $k+1$ numbers of $\left(D-\frac{k}{M}\right) T$ sub-periods is:

$$
\begin{aligned}
\Delta i_{p}^{u p}= & \left(\left(\mathcal{R}_{L}+\mathcal{R}_{C}+k \mathcal{R}_{C}\right) \frac{1-D}{D}-(M-k-1) \mathcal{R}_{C}\right) \\
& \times\left(D-\frac{k}{M}\right) \frac{(k+1) T V_{\text {OUT }}}{N^{2}} .
\end{aligned}
$$

During the $k$ numbers of $\left(\frac{k+1}{M}-D\right) T$ subperiods, $k$ phases have $V_{I N}-V_{O U T}$ across them, and $M-k$ phases have $-V_{O U T}$ across them. The per-phase current ramps down. The current ripple in each phase during the $k$ numbers of $\left(\frac{k+1}{M}-D\right) T$ subperiod is:

$$
\begin{aligned}
\Delta i_{p}^{\text {down }}= & \left(\left(\mathcal{R}_{L}+\mathcal{R}_{C}+(k-1) \mathcal{R}_{C}\right) \frac{1-D}{D}-(M-k) \mathcal{R}_{C}\right) \\
& \times\left(\frac{k+1}{M}-D\right) \frac{k T V_{\text {OUT }}}{N^{2}} .
\end{aligned}
$$

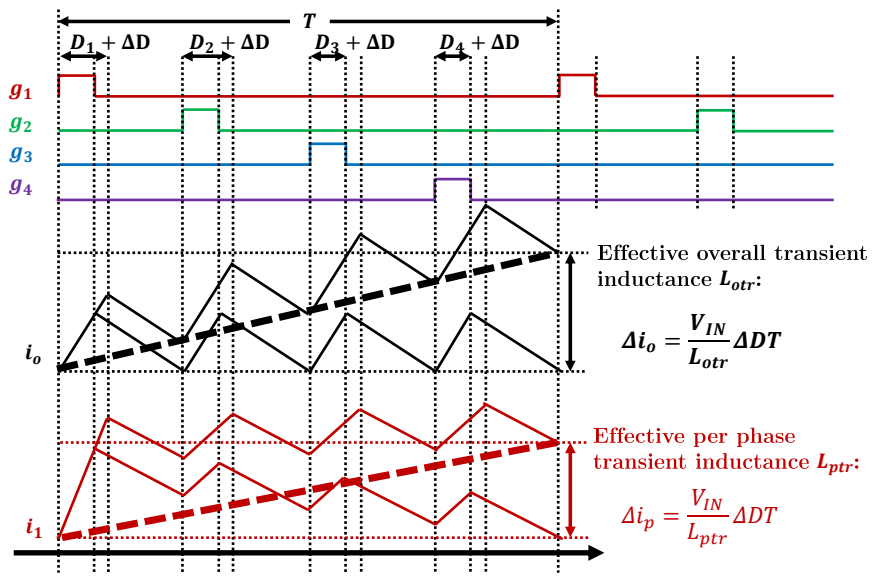

Fig. 44. Effective overall transient inductance $\left(L_{o t r}\right)$ and effective per-phase transient inductance $\left(L_{p t r}\right)$ of the multiphase coupled inductor.

The current ripple in each phase is the summation of all the ramp up and ramp down sub-periods:

$\Delta i_{p}^{s s}=\Delta i_{p}^{u p}+\Delta i_{p}^{\text {down }}=$

$\frac{T V_{O U T}}{N^{2}}\left(-\frac{k^{2} \mathcal{R}_{C}}{D M}-\frac{k \mathcal{R}_{C}}{D M}-D \mathcal{R}_{L}-D M \mathcal{R}_{C}+2 k \mathcal{R}_{C}+\mathcal{R}_{L}+\mathcal{R}_{C}\right)$

We define $L_{p s s}$ as the effective inductance of each winding in steady-state $\Delta i_{p}^{s s}=\frac{V O U T}{L_{p s s}}(1-D) T$ :

$$
L_{p s s}=\frac{N^{2}(1-D)}{-\frac{k^{2} \mathcal{R}_{C}}{D M}-\frac{k \mathcal{R}_{C}}{D M}+2 k \mathcal{R}_{C}-D M \mathcal{R}_{C}+\mathcal{R}_{C}-D \mathcal{R}_{L}+\mathcal{R}_{L}} .
$$

For an $M$-phase coupled inductor buck converter operating in continuous conduction mode with duty ratio $D$, switching period $T$, and output voltage $V_{O U T}$, the peak-to-peak current ripple of the per-phase winding current is:

$$
\Delta i_{p}=\frac{V_{O U T}(1-D) T}{L_{p s s}} .
$$

\section{Effective Overall Transient Inductance $\left(L_{o t r}\right)$}

The transient inductance determines the response of $i_{o}$ to a small perturbation in $D$. For a buck converter with a discrete inductor, the ramp up slope of the inductor current is $\frac{d i_{o}}{d t}{ }_{u p}=\frac{V_{I N}-V_{O U T}}{L}$, the ramp down slope of the inductor current is $\frac{d i_{o}}{d t}$ down $=\frac{-V_{O U T}}{L}$. If there is a small perturbation $\Delta D, i_{o}$ will ramp up $\frac{V_{I N}^{L}-V_{O U T}}{L} \Delta D T$ more, and will ramp down $\frac{V_{O U T}}{L} \Delta D T$ less. As a result, the perturbation in the inductor current is the summation of them, which is $\frac{V_{I N}}{L} \Delta D T$. In other words, the transient inductance of a single-phase uncoupled buck converter with $L$ is still $L$. For an $M$ phase coupled inductor buck converter, if $\frac{k}{M} \leq D<\frac{k+1}{M}$, the ramp up and ramp down rates are:

$$
\begin{aligned}
& \frac{d i_{o}^{t r-u p}}{d t}=\frac{\left(\mathcal{R}_{L}+M \mathcal{R}_{C}\right)(k+1-D M)}{D N^{2}} V_{O U T}, \\
& \frac{d i_{o}^{t r-d o w n}}{d t}=\frac{\left(\mathcal{R}_{L}+M \mathcal{R}_{C}\right)(k-D M)}{D N^{2}} V_{O U T} .
\end{aligned}
$$

Note that there are $M$ ripple cycles within a switching pe$\operatorname{riod} T$. A perturbation $\Delta D$ results in a perturbation of $\left(\frac{d i_{o}^{u P}}{d t}-\right.$ 
$\left.\frac{d i_{o}^{\text {down }}}{d t}\right) M T \Delta D$, which equals $\frac{\left(\mathcal{R}_{L}+M \mathcal{R}_{C}\right)}{N^{2}} V_{I N} M T \Delta D$ in the overall output current. Since

$$
\Delta i_{o}^{t r}=\frac{V_{I N}}{L_{\text {otr }}} \Delta D T=\frac{\left(\mathcal{R}_{L}+M \mathcal{R}_{C}\right)}{N^{2}} V_{I N} M \Delta D T,
$$

the effective overall transient inductance of a $M$ phase coupled inductor, regardless of the duty ratio $D$, is:

$$
L_{o t r}=\frac{N^{2}}{M\left(\mathcal{R}_{L}+M \mathcal{R}_{C}\right)} .
$$

$L_{o t r}$ is effectively connecting $M$ discrete inductors, each of reluctance value $\mathcal{R}_{L}+M \mathcal{R}_{C}$ in parallel.

\section{Effective Per-Phase Transient Inductance $\left(L_{p t r}\right)$}

Since the current equally distribute among the $M$ parallel windings, with a purtubation of $\Delta D$ in the duty ratio, the perturbation of the current in each winding is $\frac{1}{M}$ of the overall current perturbation. As a result:

$$
\frac{V_{I N}}{L_{t r w}} \Delta D T=M \frac{\left(\mathcal{R}_{L}+M \mathcal{R}_{C}\right)}{N^{2}} V_{I N} \Delta D T \times \frac{1}{M} .
$$

The effective per-phase transient inductance of a $M$ phase coupled inductor, regardless of the duty ratio $D$, is:

$$
L_{p t r}=\frac{N^{2}}{\mathcal{R}_{L}+M \mathcal{R}_{C}} .
$$

\section{ApPendix II: Model Parameter Extraction}

This Appendix introduces a method to extract parameters for the inductance-dual model from impedance measurements. By connecting an $N$-turn winding to one of the side legs and measuring the inductance, we can measure the self inductance $\left(L_{S}\right)$ of one winding:

$$
L_{S}=L_{\mu}+L_{l}=N^{2} \frac{\mathcal{R}_{L}+(M-1) \mathcal{R}_{C}}{\mathcal{R}_{L}\left(\mathcal{R}_{L}+M \mathcal{R}_{C}\right)} .
$$

By connecting all windings in parallel, we can measure the overall transient inductance $\left(L_{o t r}\right)$ of the coupled inductor:

$$
L_{\text {otr }}=\frac{L_{l}}{M}=\frac{N^{2}}{M\left(\mathcal{R}_{L}+M \mathcal{R}_{C}\right)} .
$$

$\mathcal{R}_{L}$ and $\mathcal{R}_{C}$ can be found experimentally from the measured $L_{S}, L_{o t r}$, and the known values of $N$ and $M$ :

$$
\begin{gathered}
\mathcal{R}_{L}=\frac{N^{2}(M-1)}{M\left(L_{S}-L_{o t r}\right)}, \\
\mathcal{R}_{C}=\frac{N^{2}\left(L_{S}-M L_{o t r}\right)}{M^{2} L_{o t r}\left(L_{S}-L_{o t r}\right)} .
\end{gathered}
$$

\section{REFERENCES}

[1] J. Li, C. R. Sullivan and A. Schultz, "Coupled inductor design optimization for fast-response low-voltage DC-DC converters," IEEE Applied Power Electronics Conference and Exposition, 2002.

[2] J. Li, A. Stratakos, C. R. Sullivan and A. Schultz, "Using coupled inductors to enhance transient performance of multi-phase buck converters," IEEE Applied Power Electronics Conference and Exposition, APEC 2004, pp. $1289-1293$ vol.2.

[3] A. M. Schultz and C. R. Sullivan, "Voltage converter with coupled inductive windings, and associated methods," U.S. Patent 6,362,986, March 26, 2002.

[4] T. Schmid and A. Ikriannikov, "Magnetically coupled buck converters," IEEE Energy Conversion Congress and Exposition, Denver CO, 2013.

[5] A. Ikriannikov, "The benefits of the coupled inductor technology," Tutorial 5997, Maxim Integrated, 2014.
[6] W. Chen, "Low voltage high current power conversion with integrated magnetic," Ph.D. thesis, Virginia Tech, Blacksburg, VA, Apr. 27, 1998.

[7] X. Zhou, P.-L. Wong, P. Xu, F. C. Lee and A. Q. Huang, "Investigation of candidate VRM topologies for future microproceossrs," IEEE Trans. Power Electron., vol. 15, Nov. 2000.

[8] P.-L. Wong, "Performance improment of multi-channel interleaving voltage regulator modules with integrated coupling inductors", Ph.D. thesis, Virginia Tech, Blacksburg, VA, Mar., 2001.

[9] P.-L. Wong, P. Xu, P. Yang and F. C. Lee, "Performance improvements of interleaving VRMs with coupling inductors," IEEE Trans. on Power Electron., vol. 16, no. 4, 2001.

[10] Y. Dong, "Investigation of multiphase coupled-inductor buck converters in point-of-load applications", PhD Thesis, Virginia Tech, 2009.

[11] A. V. Ledenev, G. G. Gurov and R. M. Porter, "Multiple power converter system using combining transformers", US Patent US 6,545,450 B1, April 8, 2003.

[12] P. Zumel, O. Garcia, J. A. Cobos and J. Uceda, "Tight magnetic coupling in multiphase interleaved converters based on simple transformers," IEEE Applied Power Electron. Conf., 2005.

[13] J. Czogalla, Jieli Li and C. R. Sullivan, "Automotive application of multi-phase coupled-inductor DC-DC converter," IAS Annual Meeting on Conference Record of the Industry Applications Conference, Salt Lake City, UT, USA, 2003, pp. 1524-1529, vol.3.

[14] E. Laboure, A. Cuniere, T. A. Meynard, F. Forest and E. Sarraute, "A theoretical approach to intercell transformers, application to interleaved converters," IEEE Transactions on Power Electronics, vol. 23, no. 1, pp. 464-474, Jan. 2008.

[15] K. J. Hartnett, J. G. Hayes, M. G. Egan and M. S. Rylko, "CCTT-core split-winding integrated magnetic for high-power dc-dc converters," IEEE Transactions on Power Electronics, vol. 28, no. 11, pp. 4970-4984, Nov. 2013.

[16] M. Hirakawa, M. Nagano, Y. Watanabe, K. Andoh, S. Nakatomi and S. Hashino, "High power density DC/DC converter using the close-coupled inductors," IEEE Energy Conversion Congress and Exposition, San Jose, CA, 2009, pp. 1760-1767.

[17] D. Choi, S. Baek, Y. Cho, S. Yeo and H. Kim, "Design of coupled inductor considering saturation of core by leakage inductance for a $100 \mathrm{~kW}$ interleaved inverter," 10th International Conference on Power Electronics and ECCE Asia (ICPE 2019 - ECCE Asia), Busan, Korea (South), 2019, pp. 2814-2819.

[18] Y. Liu, M. Li, Y. Dou, Z. Ouyang and M. A. E. Andersen, "Investigation and optimization for planar coupled inductor dual-phase interleaved GaNbased totem-pole PFC," IEEE Applied Power Electronics Conference and Exposition (APEC), New Orleans, LA, USA, 2020, pp. 1984-1990.

[19] S. Lu, C. Ding, Y. Mei, K. D. T. Ngo and G. Lu, "Hetero-magnetic coupled inductor (HMCI) for high frequency interleaved multiphase DC/DC converters," IEEE Applied Power Electronics Conference and Exposition (APEC), Anaheim, CA, USA, 2019, pp. 2667-2672.

[20] D. O. Boillat and J. W. Kolar, "Modeling and experimental analysis of a Coupling Inductor employed in a high performance AC power source," International Conference on Renewable Energy Research and Applications (ICRERA), Nagasaki, Japan, 2012, pp. 1-18.

[21] MIT EE Staff, Magnetic Circuits and Transformers, Cambridge MA: The MIT Press, 1943.

[22] J. G. Kassakian, M. F. Schlecht, and G. C. Verghese, "Magnetic Components," in Principles of Power Electronics, Addison-Wesley, 1991.

[23] P. T. Krein, "Concepts of Magnetics for Power Electronics," in Elements of Power Electronics, Vol. 126. New York: Oxford University Press, 1998.

[24] R. W. Erickson and D. Maksimovic, "Basic Magnetics Theory," in Fundamentals of Power Electronics, Boston, MA, USA: Kluwer, 2001.

[25] H. A. Haus and J. R. Melcher, "Introduction to Electroquasistatics and Magnetoquasistatics", in Electromagnetic Fields and Energy, Englewood Cliffs, NJ: Prentice-Hall, 1989.

[26] R. W. Erickson and D. Maksimovic, "A multiple-winding magnetics model having directly measurable parameters," Proc. of the IEEE Power Electron. Special. Conf. (PESC), vol.2, pp.1472-1478, 17-22, May 1998.

[27] S.-P. Hu, R. D. Middlebrook, and S. Cuk. "Transformer modeling and design for leakage control." Chapter 13 in Volume 2 of Advances in Switched-Mode Power Conversion.

[28] Q. Chen, F. C. Lee, J. Z. Jiang and M. M. Jovanovic, "A new model for multiple-winding transformer," Proceedings of 1994 Power Electronics Specialist Conference, Taipei, Taiwan, 1994, pp. 864-871 vol.2.

[29] S. M. Sandler, "SPICE Modeling of Magnetic Components," SwitchMode Power Supply Simulation: Designing with SPICE 3, McGraw-Hill, 2006. 
[30] E. R. Lwithwaite, "Magnetic equivalent circuits for electrical machines," Proceedings of the Institution of Electrical Engineers, vol. 114, no. 11, pp. 1805-1809, November 1967.

[31] D. C. Hamill, "Lumped equivalent circuits of magnetic components: the gyrator-capacitor approach," IEEE Transactions on Power Electronics, vol. 8, no. 2, pp. 97-103, April 1993.

[32] D. C. Hamill, "Gyrator-capacitor modeling: a better way of understanding magnetic components," IEEE Applied Power Electronics Conference and Exposition, Orlando, FL, USA, 1994, pp. 326-332.

[33] S. El-Hamamsy and E. I. Chang, "Magnetics modeling for computeraided design of power electronics circuits," IEEE Power Electronics Specialists Conference, Milwaukee, WI, USA, 1989, pp. 635-645 vol.2.

[34] G. W. Ludwig and S. El-Hamamsy, "Coupled inductance and reluctance models of magnetic components," IEEE Transactions on Power Electronics, vol. 6, no. 2, pp. 240-250, April 1991.

[35] I. G. Park and S. I. Kim, "Modeling and analysis of multi-interphase transformers for connecting power converters in parallel," IEEE Power Electronics Specialists Conference, Saint Louis, MO, USA, 1997, pp. 1164-1170, vol.2.

[36] D. J. Perreault and J. G. Kassakian, "Effects of firing angle imbalance on 12-pulse rectifiers with interphase transformers," IEEE Transactions on Power Electronics, vol. 10, no. 3, pp. 257-262, May 1995.

[37] B. Cougo, "Design and Optimization of Inter Cell Transformers for Parallel MultiCell Converters," Ph.D. Thesis, Univ. de Toulouse, 2011.

[38] F. M. Lambert, J. Mahseredjian, M. Martinez-Duro and F. Sirois, "Magnetic circuits within electric circuits: critical review of existing methods and new mutator implementations," IEEE Transactions on Power Delivery, vol. 30, no. 6, pp. 2427-2434, Dec. 2015.

[39] J. M. Alonso, G. Martinez, M. Perdigao, M. R. Cosetin and R. N. do Prado, "A systematic approach to modeling complex magnetic devices using SPICE: application to variable inductors," IEEE Transactions on Power Electronics, vol. 31, no. 11, pp. 7735-7746, Nov. 2016.

[40] V. A. Niemela, G. R. Skutt, A. M. Urling, Y.-N. Chang, T. G. Wilson, Jr., H. A. Owen and R. C. Wong, "Calculating the short-circuit impedances of a multiwinding transformer from its geometry," IEEE Power Electron. Spec. Conf., Jun. 26-29, 1989, vol. 2, pp. 607-617.

[41] M. Chen, M. Araghchini, K. K. Afridi, J. H. Lang, C. R. Sullivan and D. J. Perreault, "A systematic approach to modeling impedances and current distribution in planar magnetics," IEEE Trans. on Power Electron., vol. 31, no. 1, pp. 560-580, Jan. 2016.

[42] H. A. Owen, V. A. Niemela and T. G. Wilson, "Enhanced crosscoupled-secondaries model for multiwinding transformers," IEEE Power Electronics Specialists Conference, Toledo, Spain, 1992, pp. 1269-1276 vol.2, doi: 10.1109/PESC.1992.254729.

[43] J. G. Hayes, N. O’Donovan and M. G. Egan, "The extended T model of the multiwinding transformer," IEEE Power Electronics Specialists Conference, 2004, pp. 1812-1817.

[44] B. Hesterman, "Analysis and modeling of magnetic coupling", Denver Chapter IEEE Power Electron. Soc., 2007.

[45] C. Zhao, S. D. Round and J. W. Kolar, "An isolated three-port bidirectional DC-DC converter with decoupled power flow management," IEEE Trans. Power Electron., vol. 23, no. 5, pp. 2443-2453, Sep. 2008.

[46] Y. Chen, P. Wang, Y. Elasser and M. Chen, "Multicell reconfigurable multi-input multi-output energy router architecture," IEEE Transactions on Power Electronics, vol. 35, no. 12, pp. 13210-13224, Dec. 2020.

[47] P. Wang, Y. Chen, J. Yuan, R. C. N. Pilawa-Podgurski and M. Chen, "Differential power processing for ultra-efficient data storage," IEEE Transactions on Power Electronics, accepted.

[48] F. E. Terman, "Section 2: Circuit Elements," in Radio Engineers' Handbook, First Edition, New York, NY: McGraw-Hill Book Company, 1943.

[49] E. C. Cherry, "The duality between interlinked electric and magnetic circuits and the formation of transformer equivalent circuits," Proceedings of the Physical Society, vol. 62 part 2, section B, no. 350 B, Feb. 1949, pp. 101-111.

[50] W. H. Hayt and J. E. Kemmerly, "Inductance and Capacitance: Duality," in Engineering Circuit Analysis, Fourth Edition, New York: McGraw-Hill Book Company, 1993.

[51] L. O. Chua, Linear and Non-linear Circuits. New York: McGraw-Hill, 1987, pp. 469-472.

[52] B. Miwa, "Interleaved Conversion Techniques for High Density Power Supplies," PhD Thesis, Massachusetts Institute of Technology,1992.

[53] C. Chang and M. A. Knights, "Interleaving technique in distributed power conversion systems," IEEE Trans. on Circuits and Systems I: Fund. Theory and Applic., vol. 42, no. 5, pp. 245-251, May 1995.
[54] D. J. Perreault and J. G. Kassakian, "Distributed interleaving of paralleled power converters," IEEE Trans. on Circuits and Systems I: Fundamental Theory and Applic., vol. 44, no. 8, pp. 728-734, Aug. 1997.

[55] D. Baba, "Benefits of a Multiphase Buck Converter," Texas Instruments Analog Applications Journal, Oct. 2012.

[56] C. R. Sullivan, B. A. Reese, A. L. F. Stein and P. A. Kyaw, "On size and magnetics: Why small efficient power inductors are rare," 2016 International Symposium on 3D Power Electronics Integration and Manufacturing (3D-PEIM), Raleigh, NC, 2016, pp. 1-23.

[57] D. Zhou, Y. Elasser, J. Baek, C. R. Sullivan and M. Chen, "Inductance Dual Model and Control of Multiphase Coupled Inductor Buck Converter," IEEE Workshop on Control and Modeling of Power Electronics, Aalborg, Denmark, 2020.

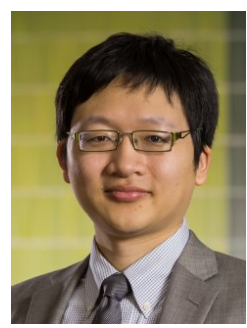

Minjie Chen (S'10-M'15-SM'20) received the B.S. degree from Tsinghua University, Beijing, China, in 2009, and the S.M., E.E., and Ph.D. degrees from Massachusetts Institute of Technology (MIT), Cambridge, MA, USA, in 2012, 2014, and 2015, respectively. He is an Assistant Professor of Electrical and Computer Engineering and Andlinger Center for Energy and the Environment at Princeton University, where he leads the Princeton Power Electronics Research Lab.

Dr. Chen received two Power Electronics Society Prize Paper Awards, a COMPEL Best Paper Award, the NSF CAREER Award, a Dimitris N. Chorafas Award for outstanding MIT Ph.D. Thesis, an Outstanding Reviewer Award from IEEE Transactions on Power Electronics, two ECCE Best Demonstration Awards, multiple APEC Outstanding Presentation Awards, a Siebel Energy Institute Research Award, a C3.ai DTI Research Award, and the First Place Award from the Innovation Forum of Princeton University. He was included in the Princeton Engineering Commendation List for Outstanding Teaching. He is the Vice Chair of IEEE PELS Technical Committee on Design Methodologies (TC10), Associate Editor of IEEE Transactions on Power Electronics, Associate Editor of IEEE Journal of Emerging and Selected Topics in Power Electronics, Associate Technical Program Committee Chair of IEEE Energy Conversion Congress and Exposition (ECCE) in 2019, and the Technical Program Committee Chair of IEEE International Conference on DC Microgrids (ICDCM) in 2021.

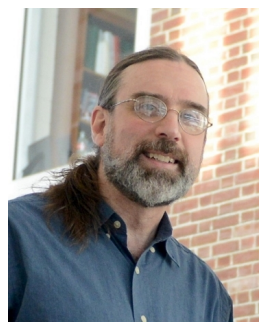

Charles R. Sullivan (S'93-M'96-SM'12-F'14) received the B.S. degree (with highest Hons.) in electrical engineering from Princeton University, Princeton, NJ, USA, in 1987, and the Ph.D. degree in electrical engineering from the University of California, Berkeley, USA, in 1996.

Between the B.S. and Ph.D. degrees, he was with Lutron Electronics designing electronic ballasts. $\mathrm{He}$ is currently a Professor at Thayer School of Engineering at Dartmouth, Hanover, NH, USA, where he is also director of the Power Management Integration Center. His research interests include modeling and design optimization of magnetics and other passive components for high-frequency power conversion and wireless power transfer.

Dr. Sullivan received the Power Electronics Society Modeling and Control Technical Achievement Award in 2018 and has received three Power Electronic Society Prize Paper Awards. He has 43 US patents and is a co-founder of Resonant Link, Inc. 